Network Working Group

Request for Comments: 1595

Category: Standards Track
T. Brown

K. Tesink

Editors

Bell Communications Research

March 1994

\title{
Definitions of Managed Objects \\ for the SONET/SDH Interface Type
}

Status of this Memo

This document specifies an Internet standards track protocol for the Internet community, and requests discussion and suggestions for improvements. Please refer to the current edition of the "Internet Official Protocol Standards" (STD 1) for the standardization state and status of this protocol. Distribution of this memo is unlimited.

Abstract

This memo defines a portion of the Management Information Base (MIB) for use with network management protocols in TCP/IP-based internets. In particular, it defines objects for managing synchronous optical Network/Synchronous Digital Hierarchy (SONET/SDH) objects. This document is a companion document with Definitions of Managed objects for the DS1/E1 and DS3/E3 Interface Types, RFC1406 [14] and RFC1407 [13].

This memo specifies a MIB module in a manner that is both compliant to the SNMPV2 SMI, and semantically identical to the peer SNMPV1 definitions.

Table of Contents

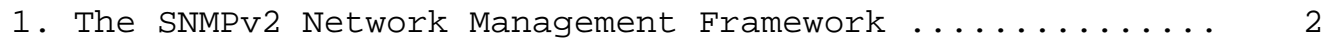

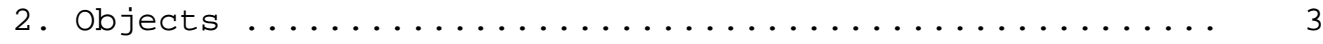

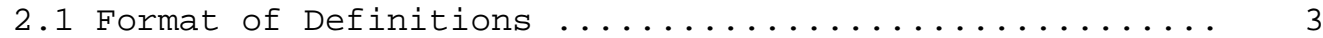

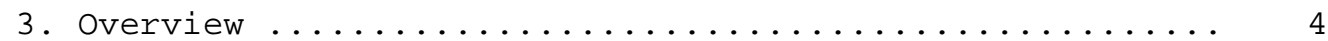

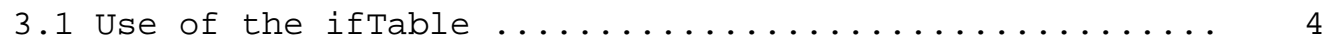

3.2 Use of ifTable for SoNET/SDH Medium/Section/Line

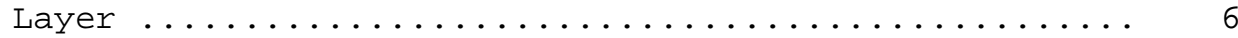

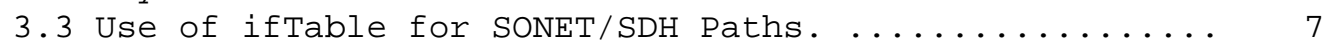

3.4 Use of iftable for SONET/SDH VTs/VCs ............ 8

3.5 SONET/SDH Terminology ...................... 9

3. 6 Values for $x$ for the Section, Line, Path, and VT

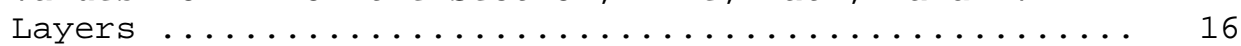

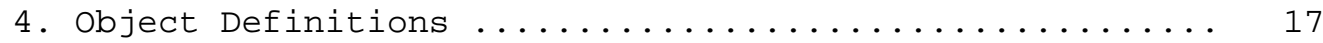

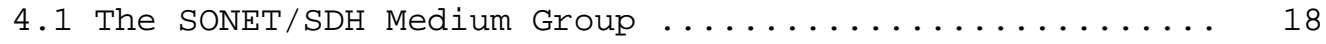




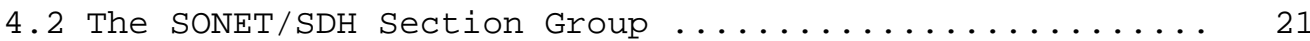

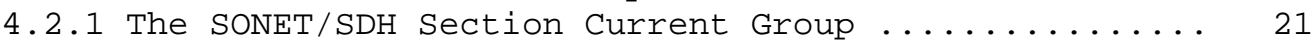

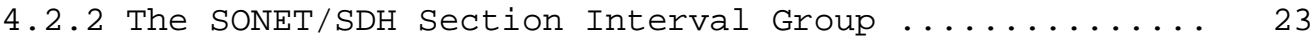

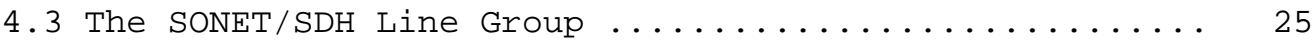

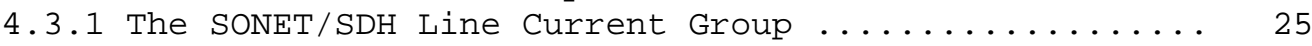

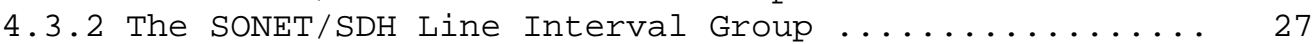

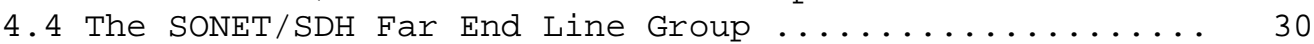

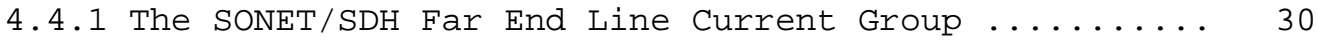

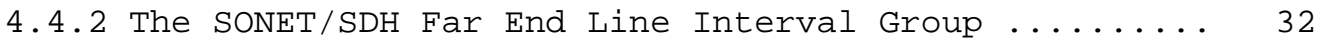

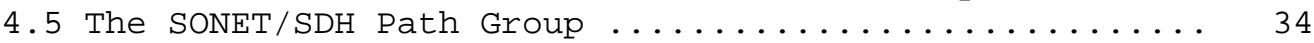

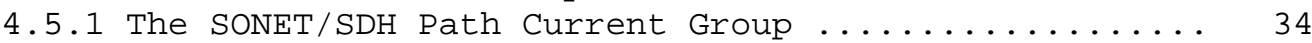

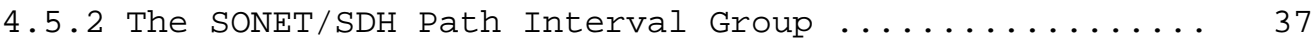

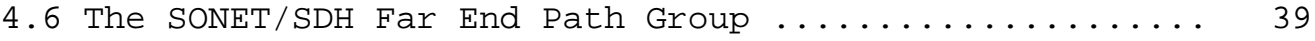

4.6.1 The SONET/SDH Far End Path Current Group ......... 39

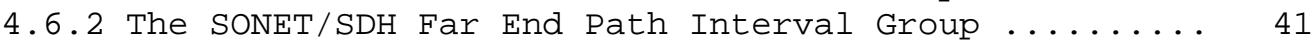

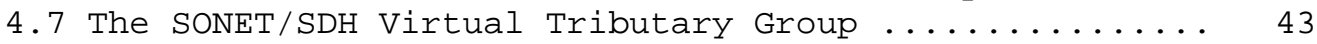

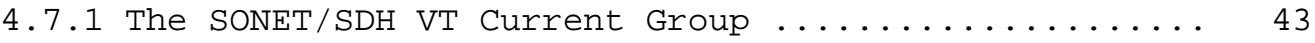

4.7.2 The SONET/SDH VT Interval Group .............. 46

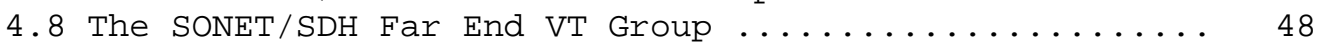

4.8.1 The SONET/SDH Far End VT Current Group ........... 48

4.8.2 The SONET/SDH Far End VT Interval Group .......... 50

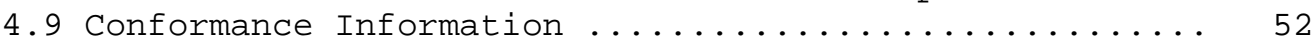

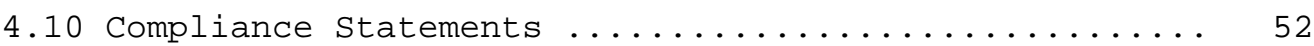

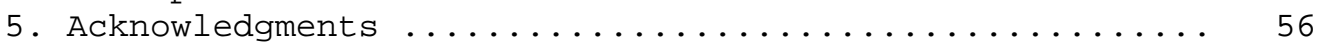

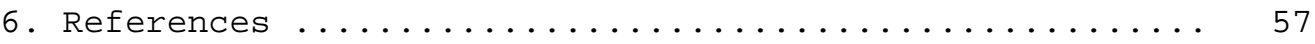

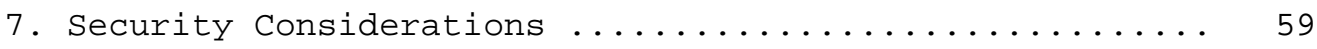

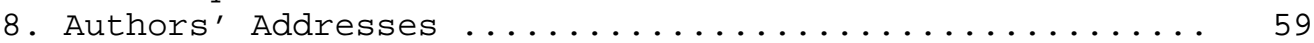

1. The SNMPv2 Network Management Framework

The SNMPv2 Network Management Framework consists of four major components. They are:

RFC 1442 [1] which defines the SMI, the mechanisms used for describing and naming objects for the purpose of management.

STD 17, RFC 1213 [6] defines MIB-II, the core set of managed objects for the Internet suite of protocols. Reference [12] defines the evolution of the Interfaces Group of MIB II in terms of extensions and precise applications of the objects.

RFC 1445 [4] which defines the administrative and other architectural aspects of the framework.

RFC 1448 [5] which defines the protocol used for network access to managed objects. 
The Framework permits new objects to be defined for the purpose of experimentation and evaluation.

This specification makes also use of:

RFC 1443 [2] which defines textual conventions for the specification of managed objects.

RFC 1444 [3] which defines conformance statements for the specification of managed objects.

2. Objects

Managed objects are accessed via a virtual information store, termed the Management Information Base or MIB. Objects in the MIB are

defined using the subset of Abstract Syntax Notation One (ASN.1) [7]

defined in the SMI. In particular, each object has a name, a syntax, and an encoding. The name is an object identifier, an administratively assigned name, which specifies an object type. The object type together with an object instance serves to uniquely identify a specific instantiation of the object. For human convenience, we often use a textual string, termed the OBJECT DESCRIPTOR, to also refer to the object type.

The syntax of an object type defines the abstract data structure corresponding to that object type. The ASN.1 language is used for this purpose. However, the SMI RFC 1442 purposely restricts the ASN.1 constructs which may be used. These restrictions are explicitly made for simplicity.

The encoding of an object type is simply how that object type is represented using the object type's syntax. Implicitly tied to the notion of an object type's syntax and encoding is how the object type is represented when being transmitted on the network. The SMI specifies the use of the basic encoding rules of ASN.1 [8], subject to the additional requirements imposed by the SNMP.

\subsection{Format of Definitions}

Section 4 contains contains the specification of all object types contained in this MIB module. The object types are defined using the conventions defined in the SMI, as amended by the extensions specified in the SNMPV2 SMI. 


\section{Overview}

These objects are used when the particular media being used to realize an interface is a SONET/SDH interface. At present, this applies to these values of the iftype variable in the Internetstandard MIB:

$$
\text { sonet (39), sonetPath (50), sonetVT (51) }
$$

The definitions contained herein are based on the SONET/SDH specifications in ANSI T1.105 and T1.106-1988 [9, 9a, 10] and CCITT $\mathrm{G} .707,708,709$, and G.783 [15-18].

\subsection{Use of the iftable}

This section specifies how the MIB II interfaces group, as defined in [12], is used for SONET/SDH interfaces. The SONET/SDH layers support several multiplexing possibilities.

For example in SONET, an Synchronous Transport Signal 3 (STS-3) has 3 SONET Paths, and a STS-3C has 1 SONET Path. Another example could be a STS-12 having 4 SONET STS-3c Paths. Similarly, a SONET Synchronous Payload Envelope (SPE) can carry many Virtual Tributaries (VTs), for example, one SONET SPE can carry $28 \mathrm{VT} 1.5 \mathrm{~s}$. It is important to note that an SPE and a VT in SONET is collectively referred to as a Virtual Container (VC) in SDH. Also, an STS is called Synchronous Transport Module (STM) in SDH.

Not all SONET/SDH equipment terminate all SONET/SDH layers. For example, an SONET/SDH STE regenerator terminates SONET/SDH Sections only, and is transparent for all layers above that. SONET/SDH AddDrop multiplexers and Digital Cross Connect systems terminate SONET/SDH Lines. SONET/SDH Terminal Multiplexers may also terminate SONET/SDH Paths and VTs/VCs.

MIB II [6], as extended by [12], accommodates these cases by appropriate use of the MIB II system group, and the interfaces group. The system group can name and describe the type of managed resource. The interfaces group defines which SONET/SDH layers apply, how these layers are configured and multiplexed. This is achieved by proper representation of SONET/SDH Layers by ifEntries as defined in [12], as follows: 


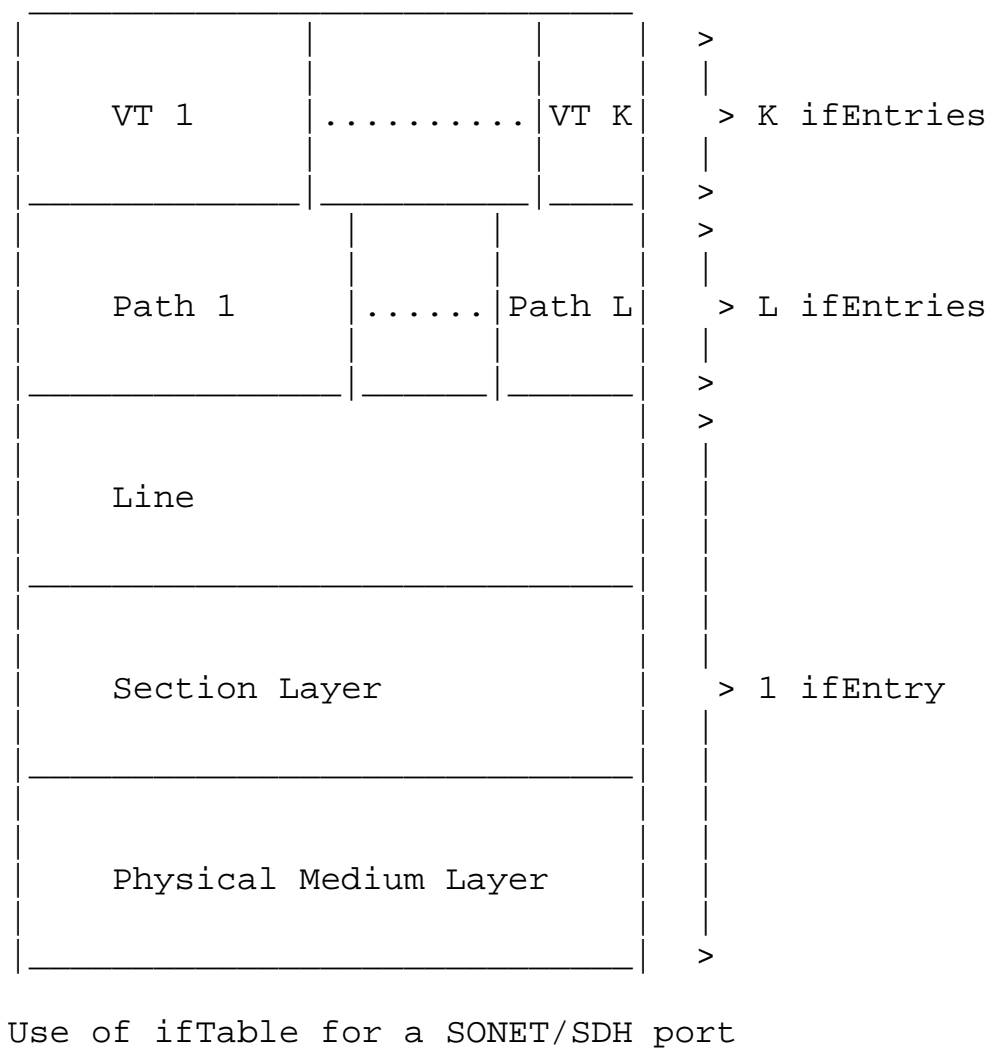

The exact configuration and multiplexing of the layers is maintained in the ifstackTable [12]. 


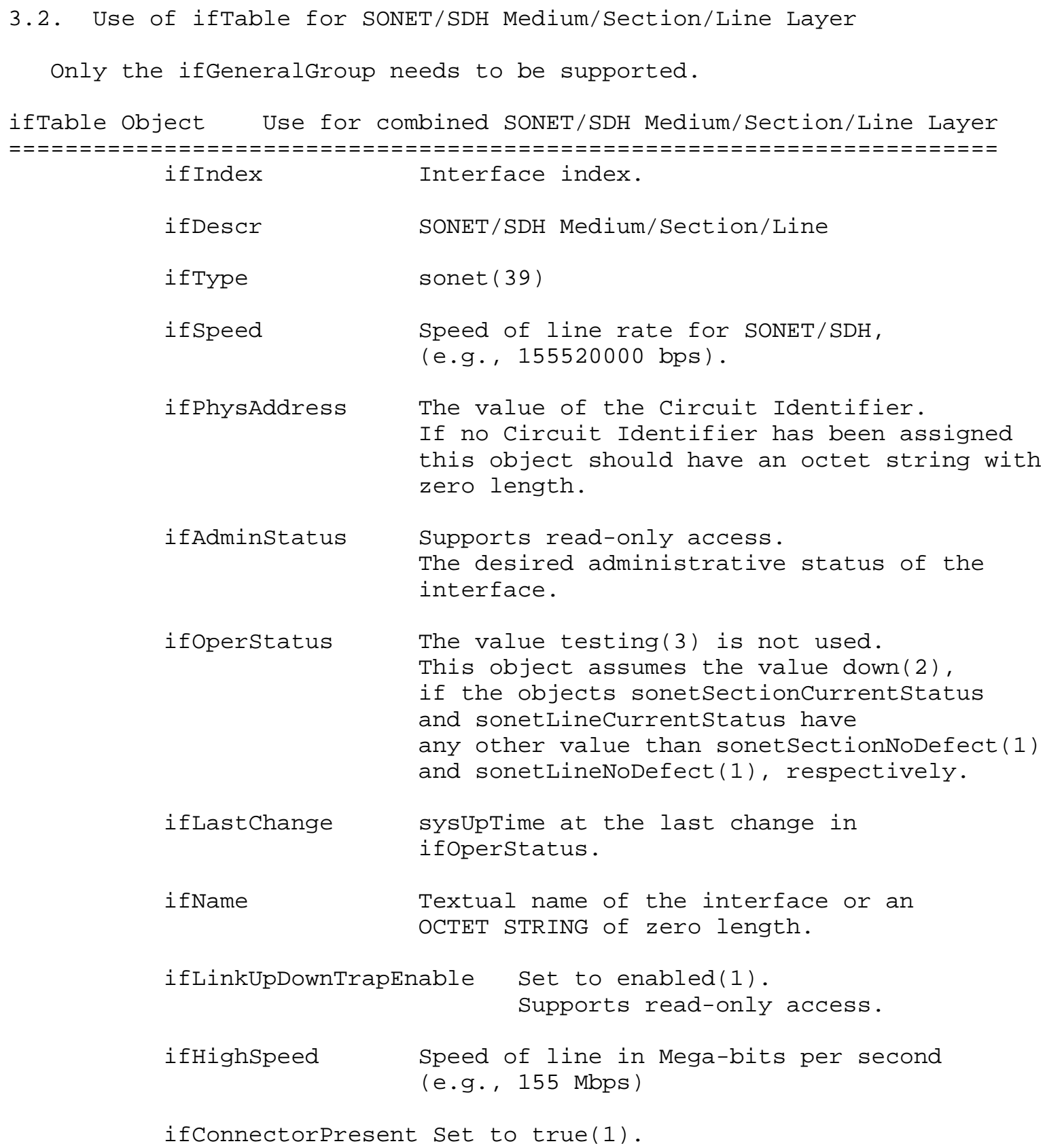


3.3. Use of ifTable for SONET/SDH Paths

Only the ifGeneralGroup needs to be supported.

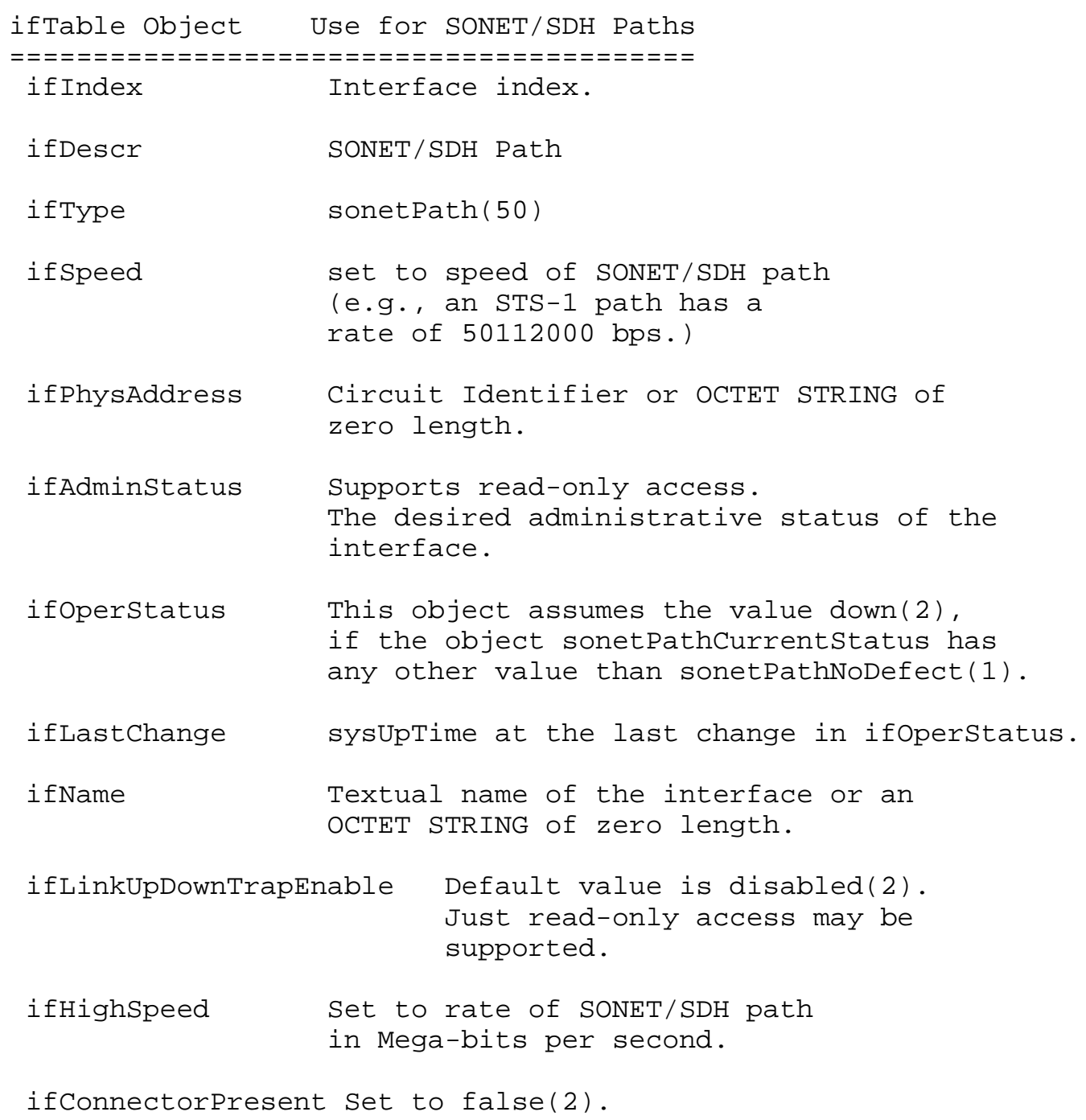


3.4. Use of ifTable for SONET/SDH VTs/VCs

Only the ifGeneralGroup needs to be supported.

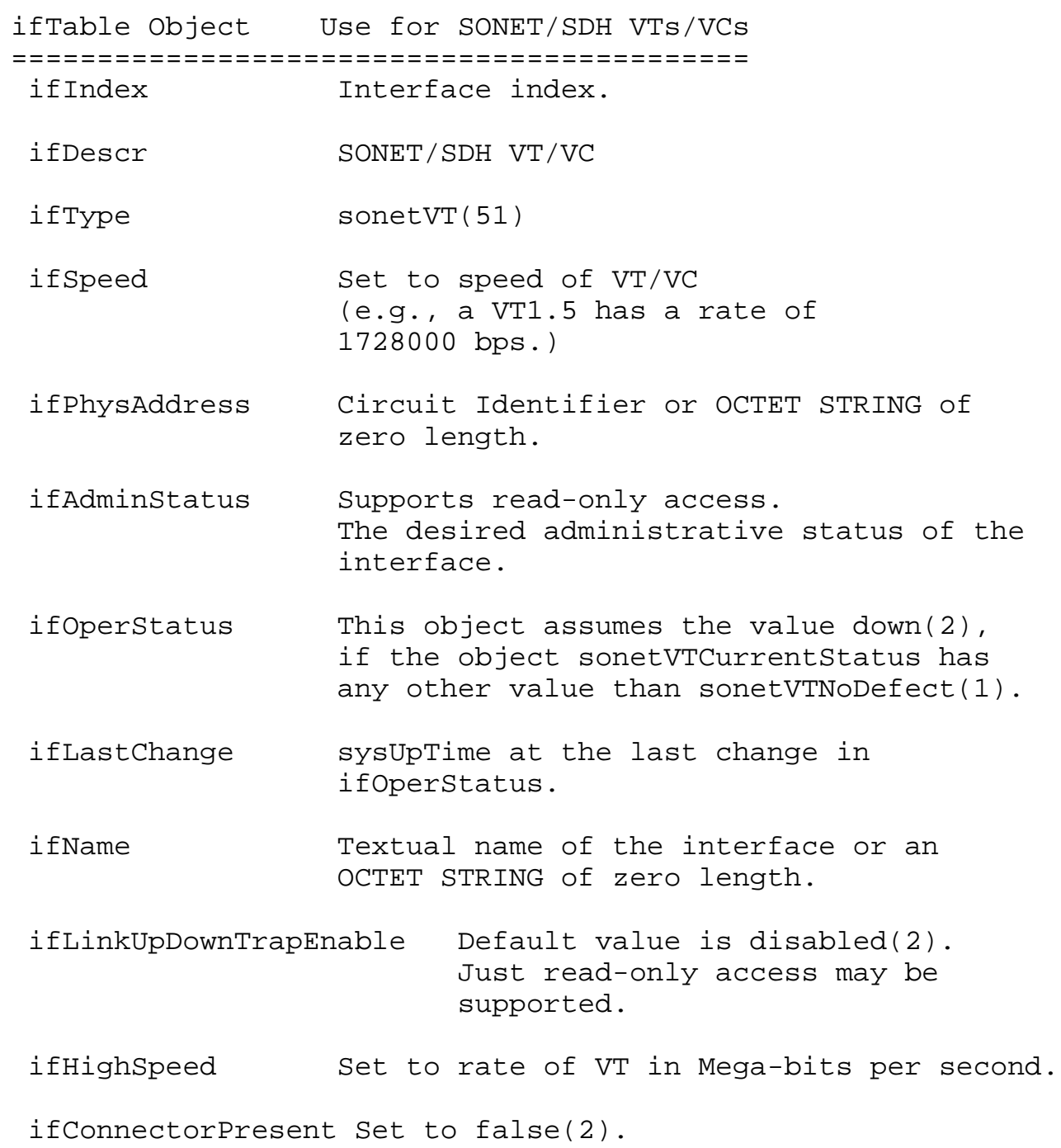




\subsection{SONET/SDH Terminology}

The terminology used in this document to describe error conditions on a SONET circuit as monitored by a SONET system are from the ANSI T1M1.3/93-005R2 [11]. The terminology used in this document to describe error conditions on a SDH circuit as monitored by a SDH system are from the CCITT G.783 [18]. Only the SONET Performance Monitoring terminology is defined in this document. The definitions for SDH Performance Monitoring terms are similar but not identical, and they can be found in [18]. If the definition in this document does not match the definition in the ANSI T1M1.3/93-005R2 draft document, the implementer should follow the definition described in this document.

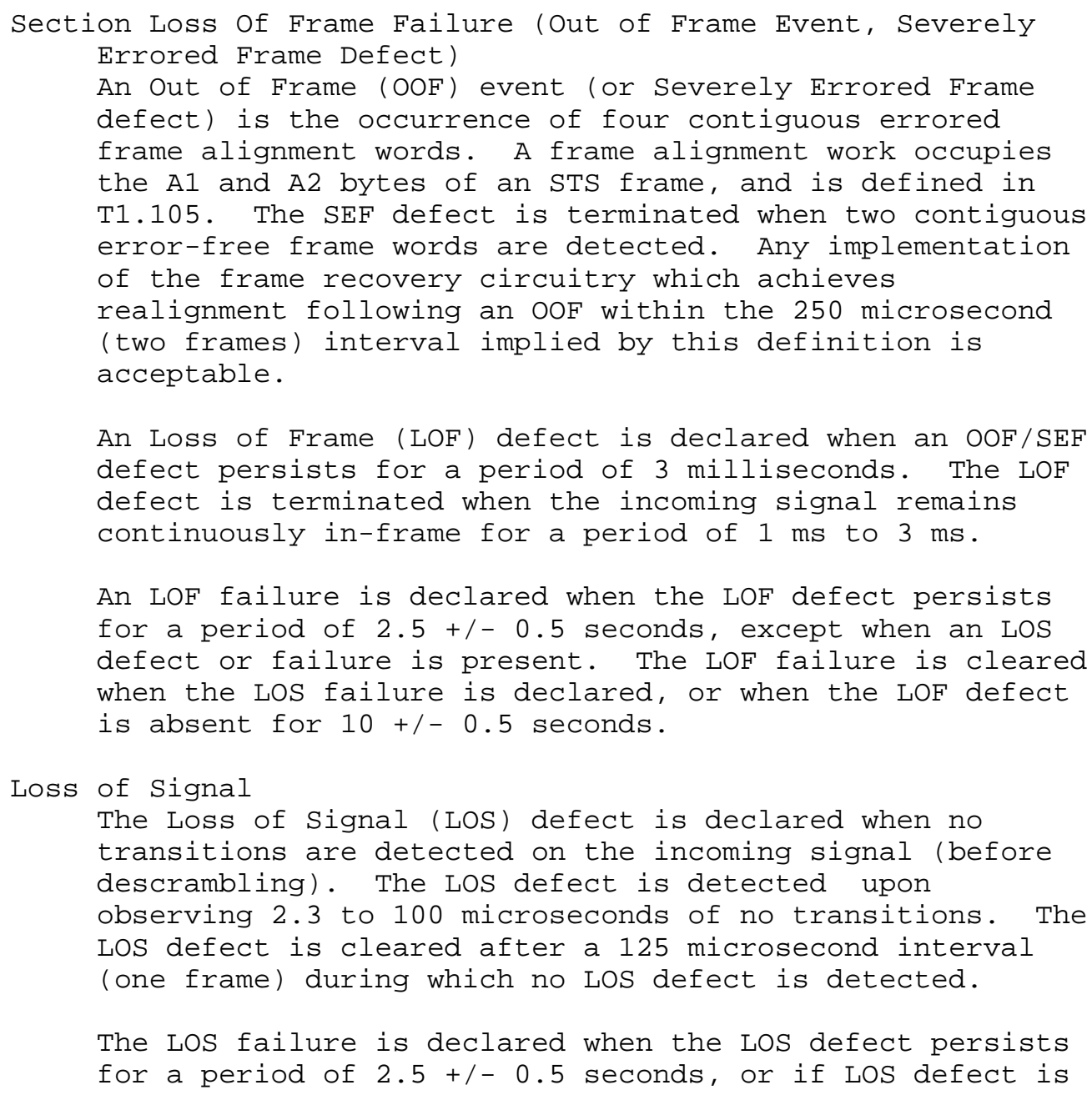


present when the criteria for LOF failure declaration have been met. The LOS failure is cleared when the LOS defect is absent for a period of $10+/-0.5$ seconds. Declaration of LOS failure clears any existing LOF failure. Clearing the Los failure allows immediate declaration of the LOF failure if conditions warrant.

STS-Path Loss of Pointer An Loss of Pointer (LOP) defect is declared when either a valid pointer is not detected in eight consecutive frames, or when eight consecutive frames are detected with the New Data Flag (NDF) set to "1001" without a valid concatenation indicator (see ANSI T1.105). A LOP defect is terminated when either a valid pointer with a normal NDF set to "0110", or a valid concatenation indicator is detected for three contiguous frames. Incoming STS-Path AIS shall not result in the declaration of a LOP defect.

A STS-Path LOP failure is declared when the STS-Path LOP defect persists for a period of $2.5+/-0.5$ seconds. A STS-Path LOP failure is cleared when the STS-Path LOP defect is absent for $10+/-0.5$ seconds.

VT Loss of Pointer

A VT LOP defect is declared when either a valid pointer is not detected in eight consecutive VT superframes, or when eight consecutive VT superframes are detected with the NDF set to "1001" without a valid concatenation indicator. A VT LOP defect is terminated when either a valid pointer with a normal NDF set to "0110", or a valid concatenation indicator is detected for three contiguous VT superframes. Incoming VT-Path AIS shall not result in declaring a VT LOP defect.

A VT LOP failure is declared when the VT LOP defect persists for $2.5+/-0.5$ seconds. A VT LOP failure is cleared when the VT LOP defect is absent for $10+/-0.5$ seconds.

Line Alarm Indication Signal

A Line Alarm Indication Signal (L-AIS) is defined in ANSI T1.105. The following criteria are specific to the L-AIS defect:

-- Line AIS defect is detected as a "111" pattern in bits 6,7 , and 8 of the $\mathrm{K} 2$ byte in five consecutive frames. 
-- Line AIS defect is terminated when bits 6, 7, and 8 of the K2 byte do not contain the code "111" for five consecutive frames.

A Line AIS failure is declared when the Line AIS defect persists for a period of $20.5+/-0.5$ seconds. A Line AIS failure is cleared when the Line AIS defect is absent for $10+/-0.5$ seconds.

STS-Path Alarm Indication Signal

The STS-Path Alarm Indication Signal (AIS) is defined in ANSI T1.105 as all ones in bytes H1, H2, and H3 as well as all ones in the entire STS SPE. The following criteria are specific to the STS-Path AIS defect:

-- STS-Path AIS defect is detected as all ones in bytes $\mathrm{H} 1$ and $\mathrm{H} 2$ in three contiguous frames.

-- The STS-Path AIS defect is terminated when a valid STS Pointer is detected with the NDF set to "1001" (inverted) for one frame, or "0110" (normal) for three contiguous frames.

A STS-Path AIS failure is declared when the STS-Path AIS defect persists for $2.5+/-0.5$ seconds. A STS-Path AIS failure is cleared when the STS-Path AIS defect is absent for $10+/-0.5$ seconds.

VT-Path Alarm Indication Signal The VT-Path Alarm Indication Signal (AIS) is only applicable for VTs in the floating mode of operation. VT-Path AIS is used to alert the downstream VT Path Terminating Entity (PTE) of an upstream failure. Upon detection of a failure, Line AIS, or STS-Path AIS, an STS PTE will generate downstream VT-Path AIS if the STS Synchronous Payload Envelope (SPE) is carrying floating VTs. VT-Path AIS is specified in ANSI T1.105 as all ones in bytes V1, V2, V3, and V4, as well as all ones in the entire VT SPE. The following criteria are specific to VT-Path AIS defect:

-- VT-Path AIS defect is detected by a VT PTE as all ones in bytes V1 and V2 in three contiguous VT superframes.

-- VT-Path AIS defect is terminated when valid VT pointer with a valid VT size is detected with the NDF set to "1001" (inverted) for one VT superframe, or "0110" 
(normal) for three contiguous VT superframes are detected.

A VT-Path AIS failure is declared when the VT-Path AIS defect persists for $2.5+/-0.5$ seconds. A VT-Path AIS failure is cleared when the VT-Path AIS defect is absent for $10+/-0.5$ seconds.

Line Remote Defect Indication

Line Remote Defect Indication (RDI) (aka Line FERF) signal is the occurrence of a "110" pattern in bit positions 6, 7, and 8 of the K2 byte in STS-1 \#1 of the STS-N signal. Line RDI is defined in ANSI T1.105. The following criteria are specific to Line RDI defect:

-- Line RDI defect is a "110" code in bits 6, 7, and 8 of the K2 byte of in STS-1 \#1 in five consecutive frames.

-- Line RDI defect is terminated when any code other than "110" is detected in bits 6, 7, and 8 of the k2 byte in five consecutive frames.

A Line Remote Failure Indication (RFI) failure is declared when the incoming Line RDI defects lasts for 2.5 $+/-0.5$ seconds. The Line RFI failure is cleared when no Line RDI defects are detected for $10+/-0.5$ seconds.

STS-Path Remote Defect Indication STS-Path RDI (aka STS-Path FERF) signal shall be generated within 100 milliseconds by the STS PTE upon detection of an AIS or LOP defect. Transmission of the STS-Path RDI signal shall cease within 100 milliseconds when the STS PTE no longer detects STS-Path AIS or STSPath LOP defect. The STS-Path RDI shall accurately report the presence or absence of STS-Path AIS or STSPath LOP defects. STS-Path RDI defect is defined in ANSI T1.105. The following requirements are specific to the STS-Path RDI defect:

-- STS-Path RDI is detected by all STS PTEs. STS-Path RDI is detected by the upstream STS PTE as a "I" in bit five of the Path status byte (G1) for five contiguous frames.

-- Removal of STS-Path Remote Defect Indication is detected by a "0" in bit 5 of the G1 byte in five contiguous frames. 
A STS-Path Remote Failure Indication (RFI) failure is declared when the incoming STS-Path RDI defects lasts for $2.5+/-0.5$ seconds. The STS-Path RFI failure is cleared when no STS-Path RDI defects are detected for $10+/-0.5$ seconds.

VT-Path Remote Defect Indication VT Path RDI (aka VT Path FERF) signal shall be generated within 100 milliseconds by the VT PTE upon detection of a VT-Path AIS or LOP defect. Transmission of the VT-Path RDI signal shall cease within 100 milliseconds when the VT PTE no longer detects VT-Path AIS or VT-Path LOP defect. The VT-Path RDI shall accurately report the presence or absence of VT-Path AIS or VT-Path LOP defects. VT-Path RDI defect is defined in ANSI T1.105. The following requirements are specific to VT-Path RDI defect:

-- VT-Path RDI defect is the occurrence of a "1" in bit 4 of the VT-Path Overhead byte (V5) in five contiguous frames.

-- VT-Path RDI defect is terminated when a "0" is detected in bit 4 of the VT-Path Overhead byte (V5) for five contiguous frames.

A VT-Path Remote Failure Indication (RFI) (derived) failure is declared when the incoming VT-Path RDI defects lasts for $2.5+/-0.5$ seconds. The VT-Path RFI failure is cleared when no VT-Path RDI defects are detected for $10+/-0.5$ seconds.

VT-Path Remote Failure Indication The VT-Path RFI signal is only required for the case of byte synch mapped DS1s where the DS1 frame bit is not mapped. The VT-Path RFI is specified in ANSI T1.105, where it is currently called VT path yellow. When provided, the VT-Path RFI signal is used to indicate the occurrence of far-end failures. When the VT-Path RFI is not provided, far-end failures are derived from local timing of the VT-Path RDI defect. The VT-Path RFI failure is declared within $5 \mathrm{~ms}$ of detecting the incoming VT-Path RFI Signal. The VT-Path Remote Failure Indication (RFI) failure is cleared within $50 \mathrm{~ms}$ of detecting the removal of the incoming VT-Path RFI signal. 
Coding Violation Coding Violations (CV) are Bit Interleaved Parity (BIP) errors that are detected in the incoming signal. CV counters are incremented for each BIP error detected. That is, each BIP-8 can detect up to eight errors per STS-N frame, with each error incrementing the CV counter. Section CVs shall be collected using the BIP-8 in the B1 byte located in the section Overhead of STS-1 \#1. Line CVs shall be collected using the BIP-8s in B2 bytes located in the Line Overhead of each STS-1 (since all CVs on an STS-N line are counted together, this is equivalent to counting each error in the $\mathrm{BIP}-8{ }^{*} \mathrm{~N}$ contained in the B2 bytes of the STS-N Line Overhead). Thus, on an STS-N signal, up to $8 \mathrm{x} \mathrm{N}$ CVs may occur in each frame. Path CVs shall be collected using the BIP-8 in the B3 byte of the STS-Path Overhead of the STS SPE. VT CVs shall be collected using the BIP-2 in the V5 overhead byte of the floating VT.

Errored Seconds At each layer, an Errored Second (ES) is a second with one or more Coding Violations at that layer OR one or more incoming defects (e.g., SEF, LOS, AIS, LOP) at that layer has occurred.

Severely Errored Seconds At each layer, an Severely Errored Second (SES) is a second with $\mathrm{x}$ or more CVs at that layer, or a second during which at least one or more incoming defects at that layer has occurred. Values of $x$ vary depending on the line rate and the Bit Error Rate. See Section 3.4 for values for $x$.

Severely Errored Framing Seconds A Severely Errored Framing Second (SEFS) is a seconds with containing one or more SEF events. This counter is only counted at the section Layer.

Unavailable Seconds At the Line, Path, and VT layers, an unavailable second is calculated by counting the number of seconds that the interface is unavailable. At each layer, the SONET/SDH interface is said to be unavailable at the onset of 10 contiguous SESS. The $10 \mathrm{SESs}$ are included in unavailable time. Once unavailable, the SONET/SDH interface becomes available at the onset of 10 contiguous seconds with no SESS. The 10 seconds with no SESs are excluded from unavailable time. With respect to the SONET/SDH error 
counts at each layer, all counters at that layer are incremented while the SONET/SDH interface is deemed available at that layer. While the interface is deemed unavailable at that layer, the only count that is incremented is UASs at that layer.

A special case exists when the 10 or more second period crosses the 900 second statistics window boundary, as the foregoing description implies that the SES and UAS counters must be adjusted when the Unavailable Signal State is entered. Clearly, successive GETs of the affected sonetPathIntervalSES and sonetPathIntervalUAS (for the Line and VT also) objects will return differing values if the first GET occurs during the first few seconds of the window. This is viewed as an unavoidable side-effect of selecting the presently defined managed objects as a basis for this memo.

Unequipped

If a Path or VT connection is not provisioned (idle) the SONET equipment will signal this state by transmitting the Path or VT Signal Label as follows:

- byte C2 of the STS Path Overhead equal to 0 for an unequipped Path,

- byte V5 of the VT Path Overhead equal to 0 for an unequipped VT.

Signal Label Mismatch

A Path or VT connection is not correctly provisioned if a received Path or VT Signal Label mismatch occurs. A received Signal Label is considered mismatched if it does not equal either the locally provisioned value or the value 'equipped non-specific' (1 hex). Note that any received non-zero Signal Label is considered a locally provisioned value of 'equipped non-specific'. Only inservice, provisioned Path Terminating equipment can detect mismatched Signal labels. It is considered provisioned if it has been configured for a mapping and has been assigned signals to and from which the mapping takes place.

Circuit Identifier

This is a character string specified by the circuit vendor, and is useful when communicating with the vendor during the troubleshooting process. 
3.6. Values for $\mathrm{x}$ for the Section, Line, Path, and VT Layers

Value for $x$ for SONET/SDH Section SES Definition

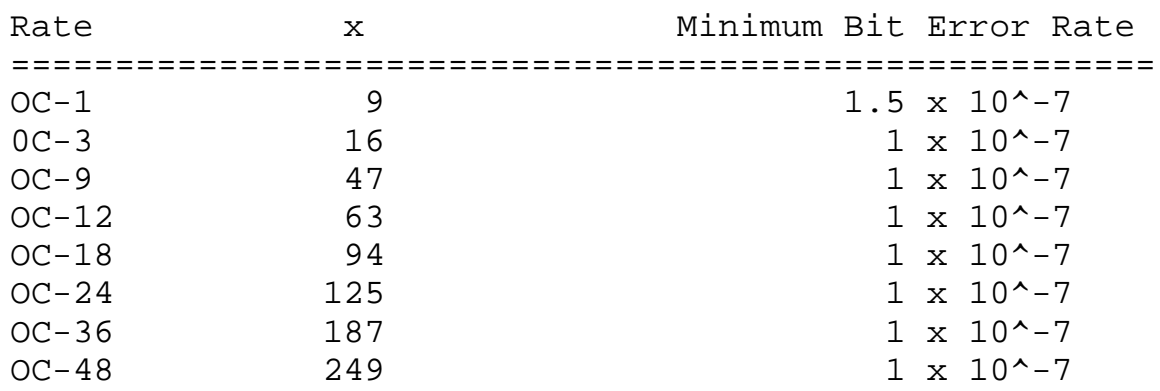

Value for $x$ for SONET/SDH Line SES Definition

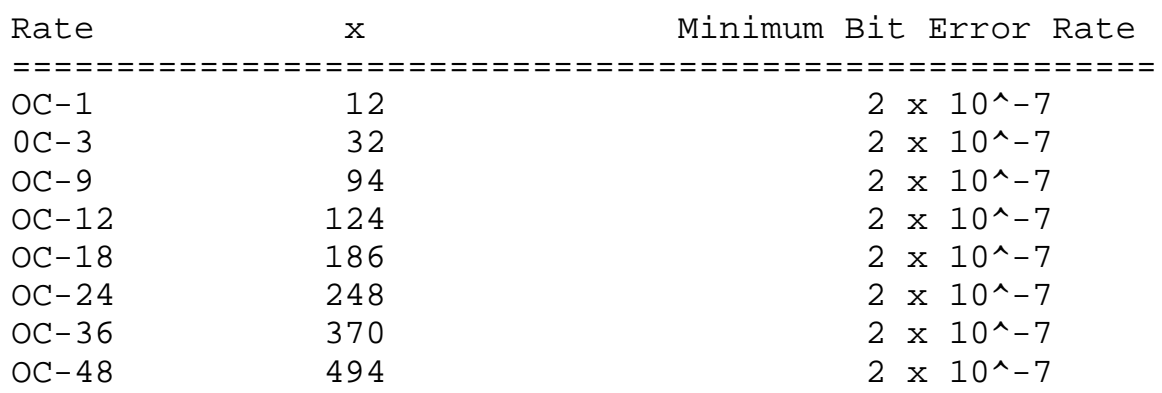

Value for $x$ for SONET/SDH STS-Path SES Definition

$\begin{array}{lcr}\text { Rate } & x & \text { Minimum Bit Error Rate } \\ ======================================= \\ \text { STS }-1 & 9 & 1.5 \times 10^{\wedge}-7 \\ \text { STS }-3 & 16 & 1 \times 10^{\wedge}-7\end{array}$

Value for $x$ for SONET/SDH VT-Path SES Definition

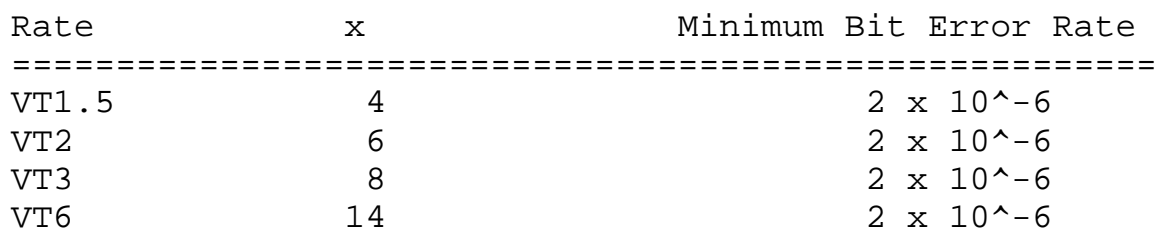


4. Object Definitions

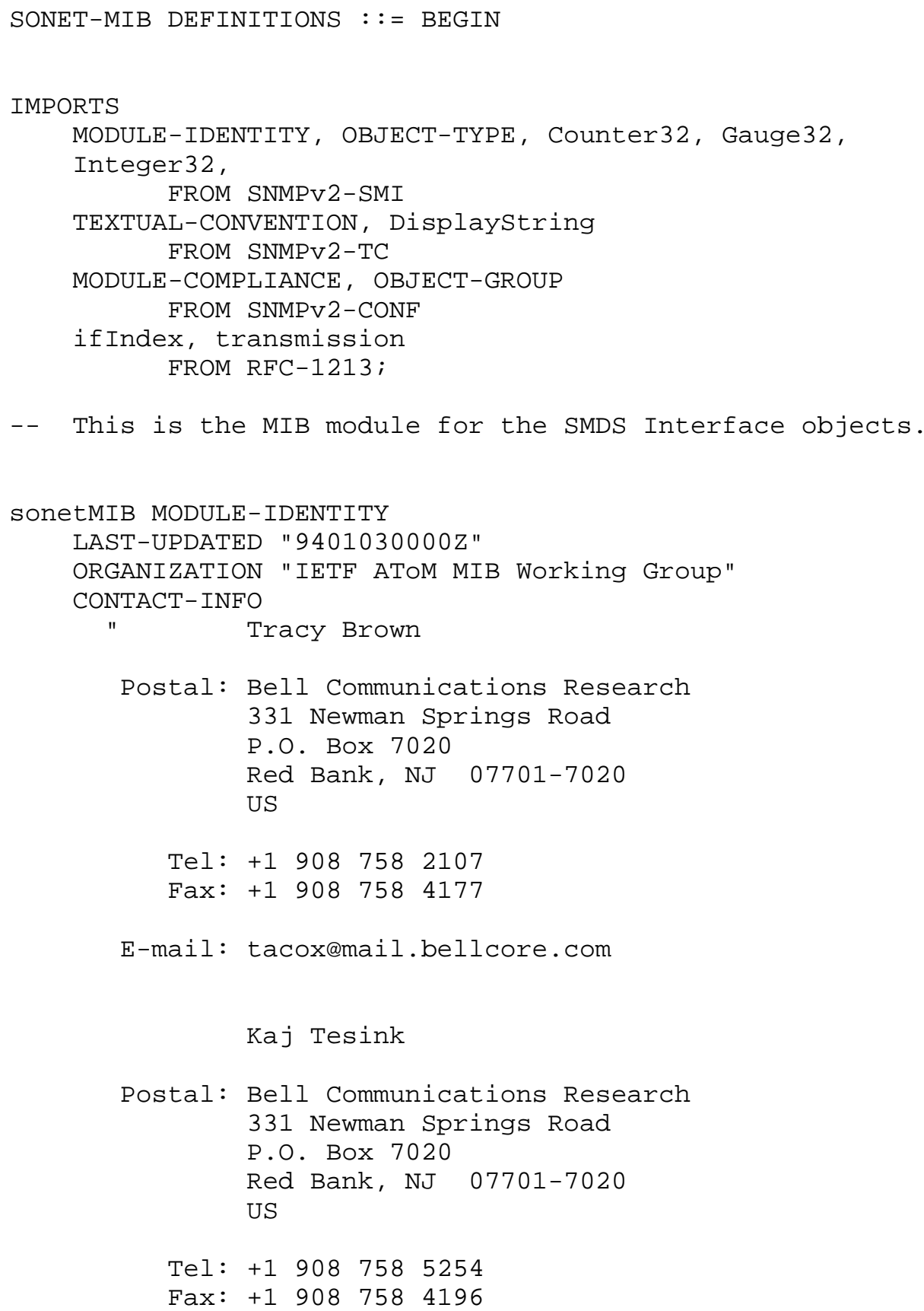




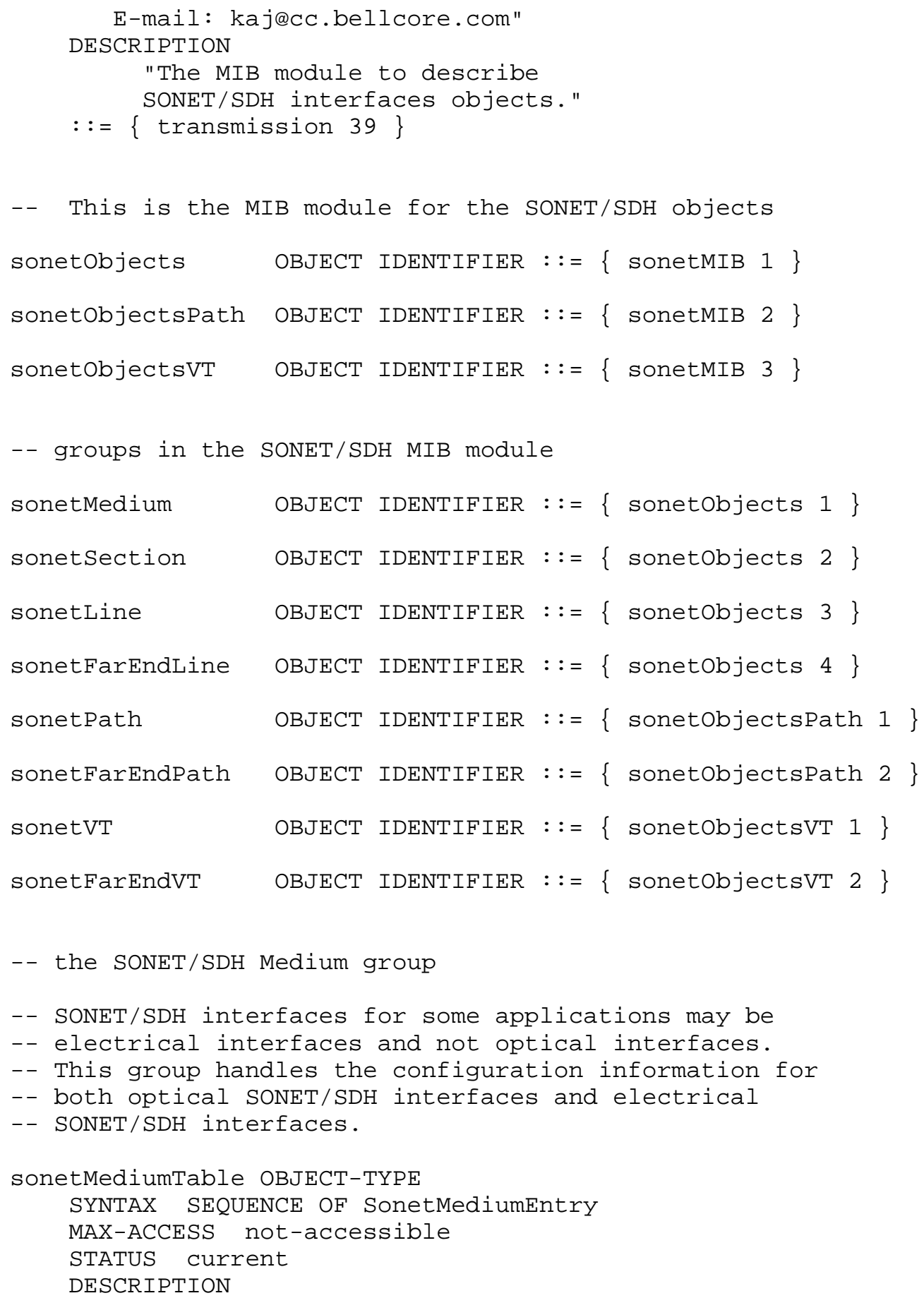




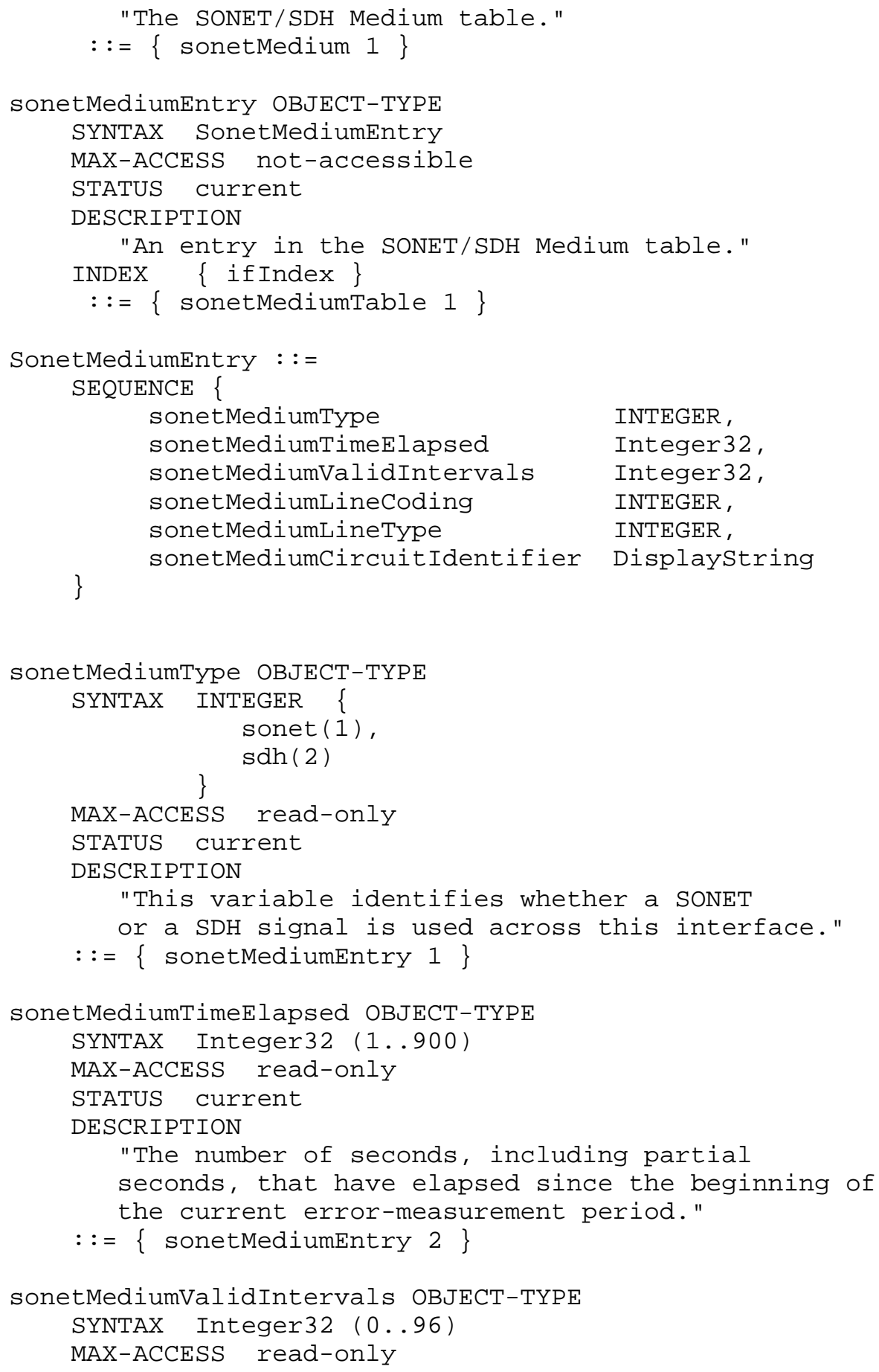




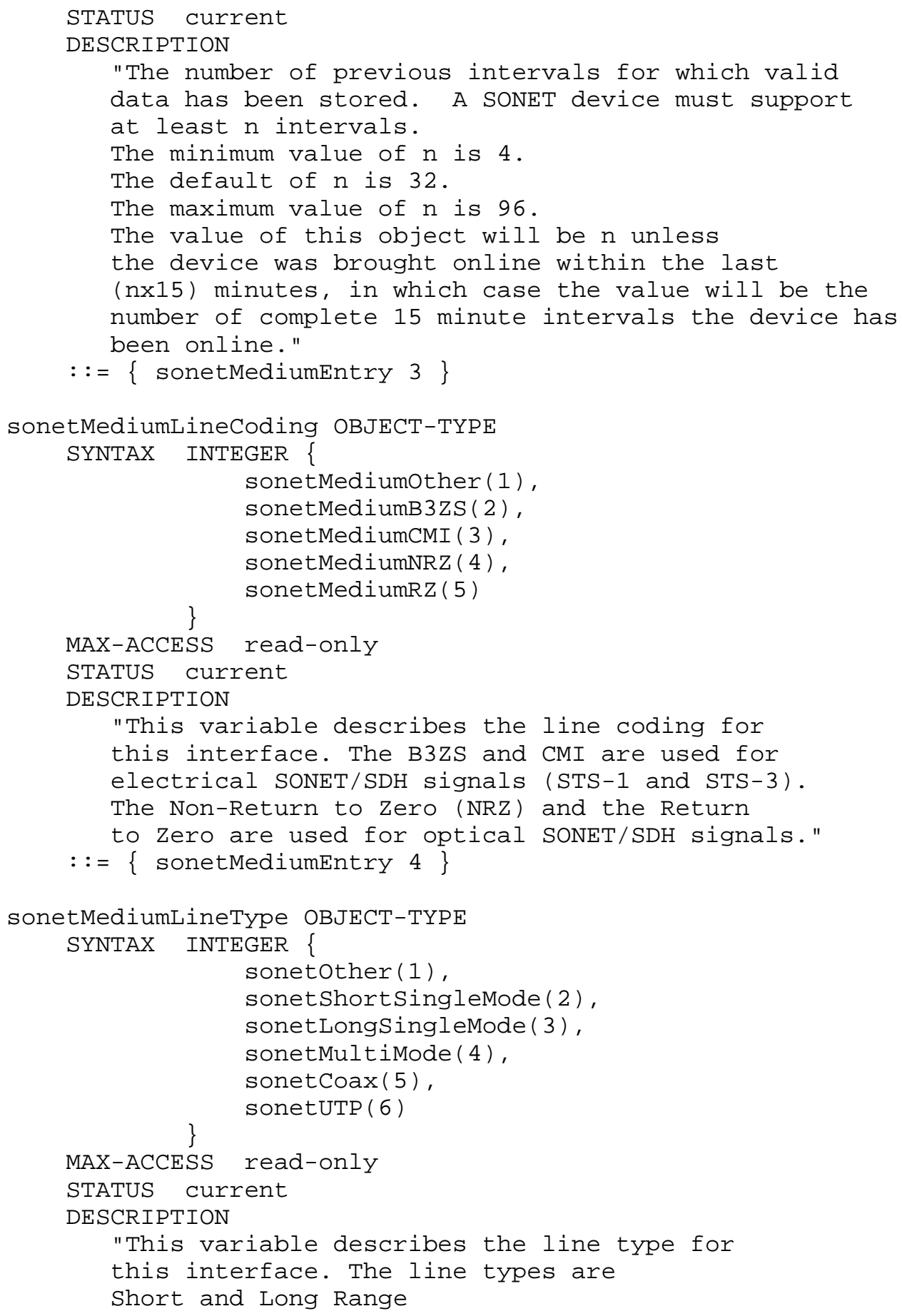




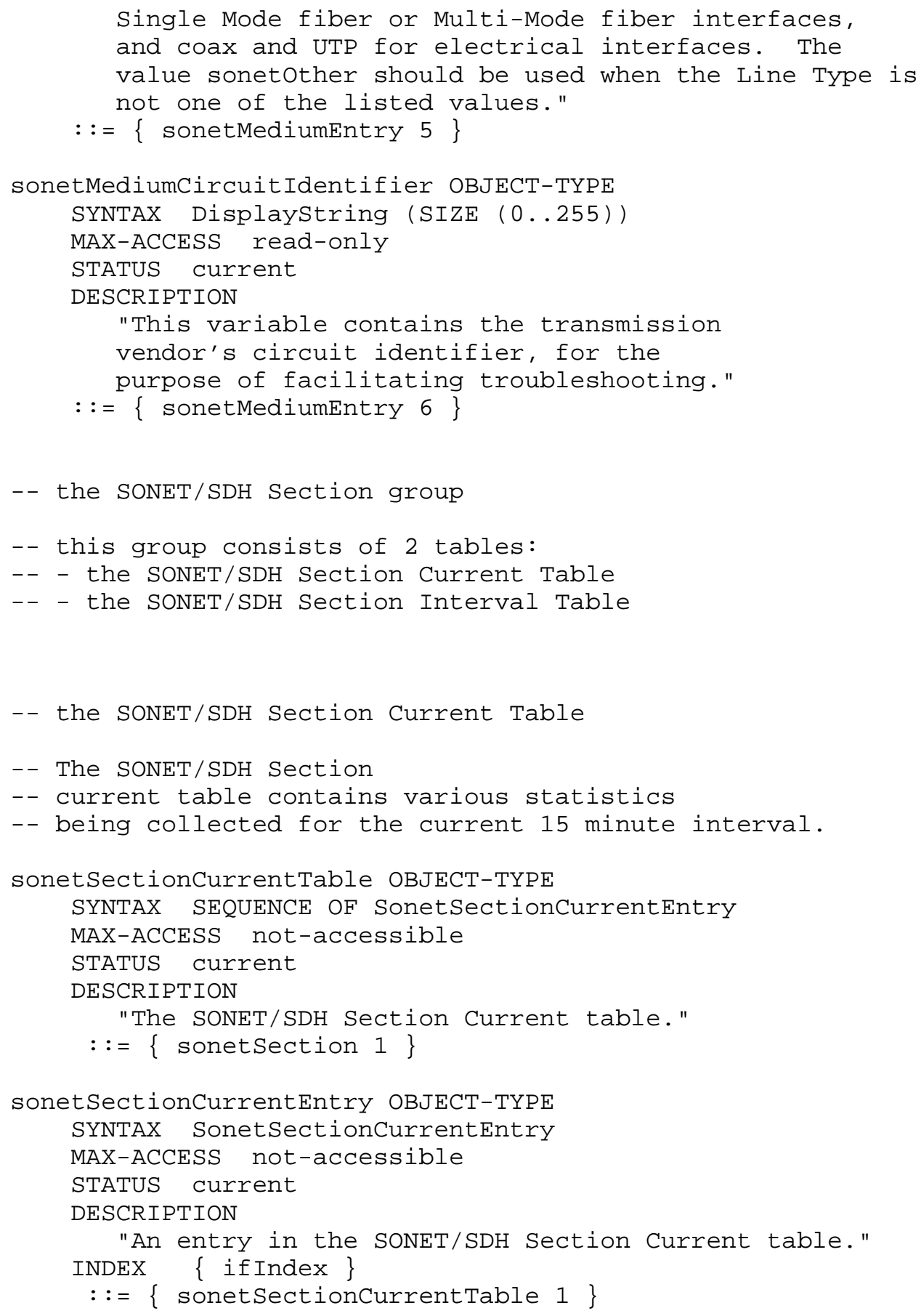




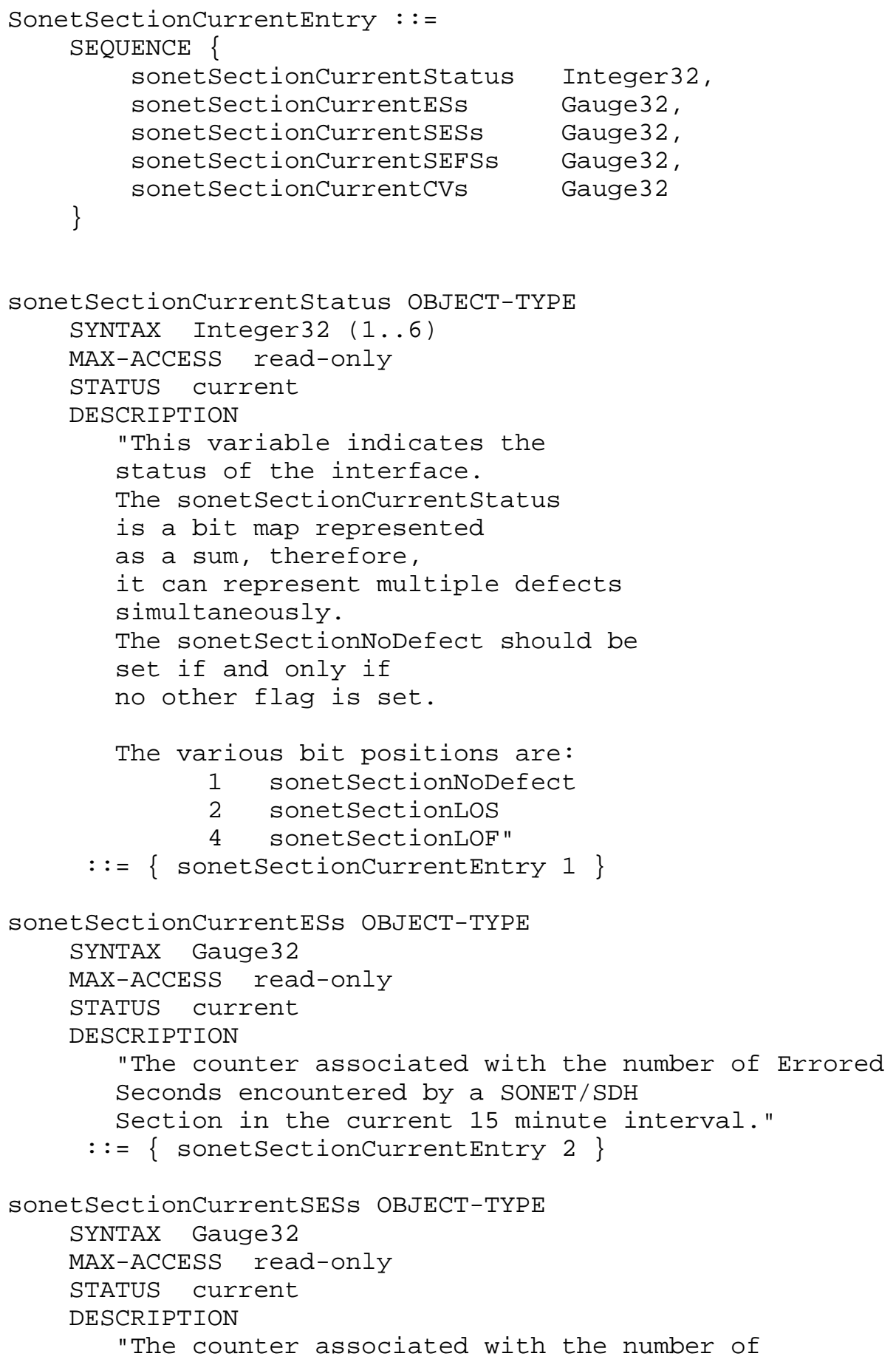

"The counter associated with the number of 


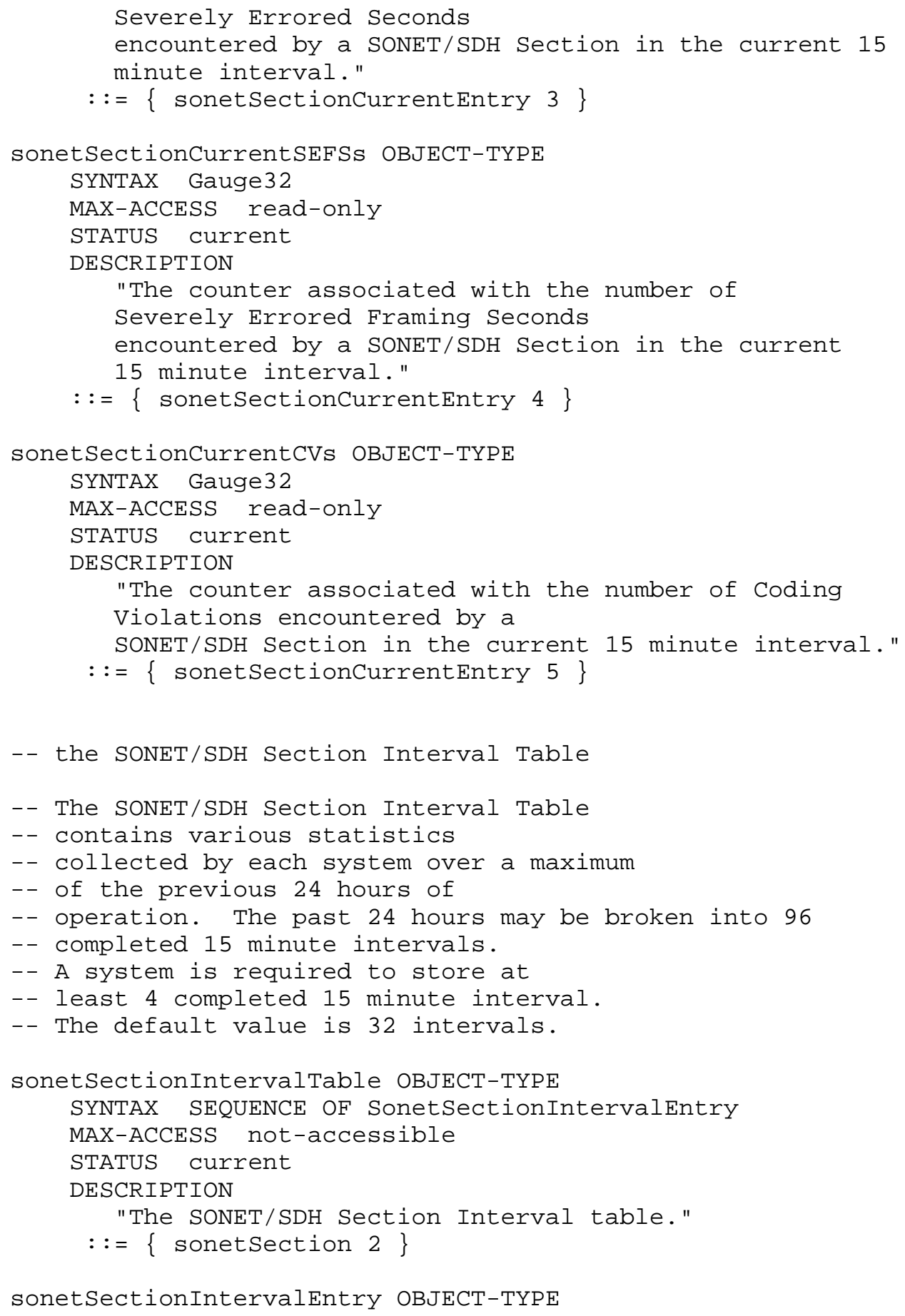




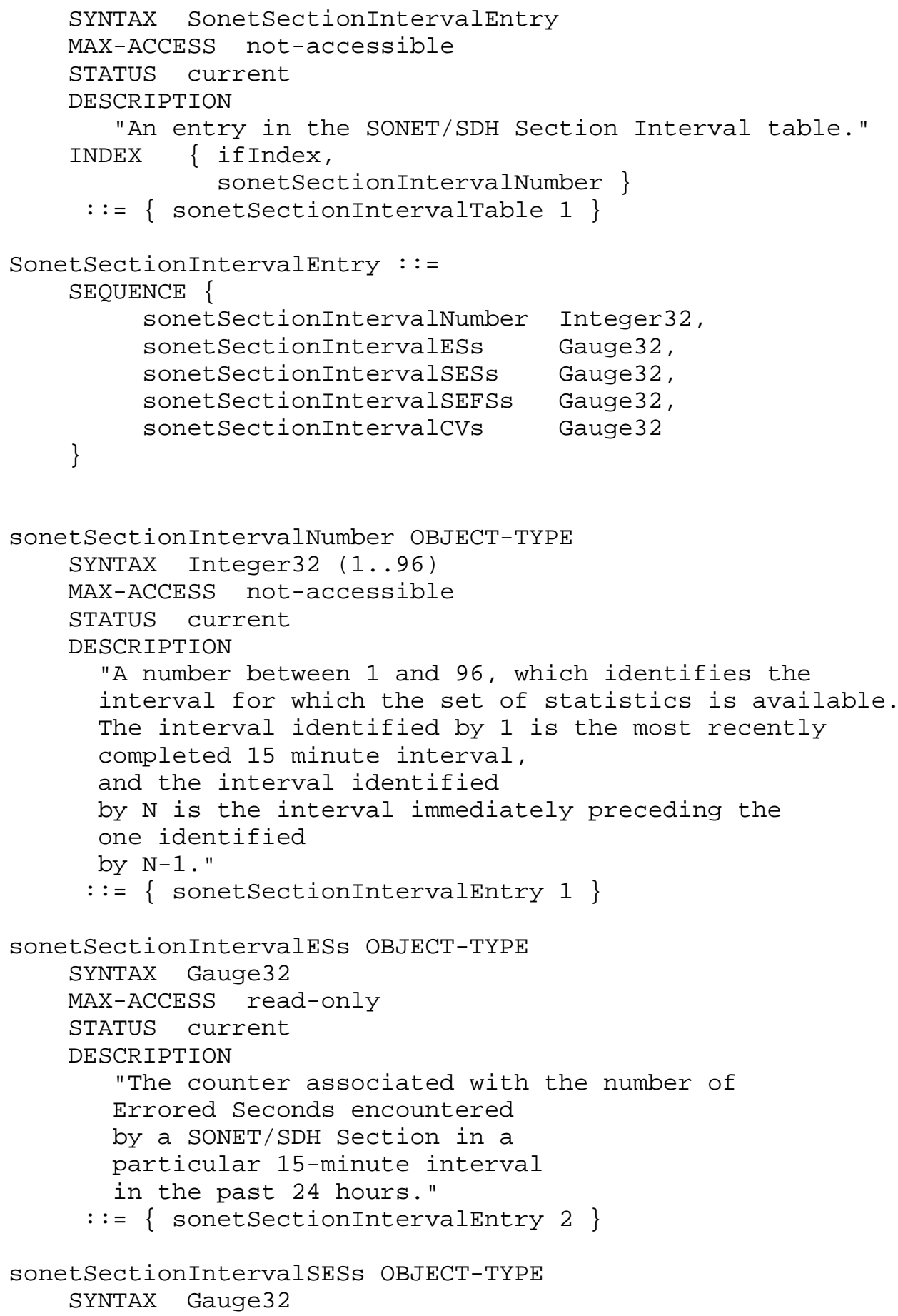




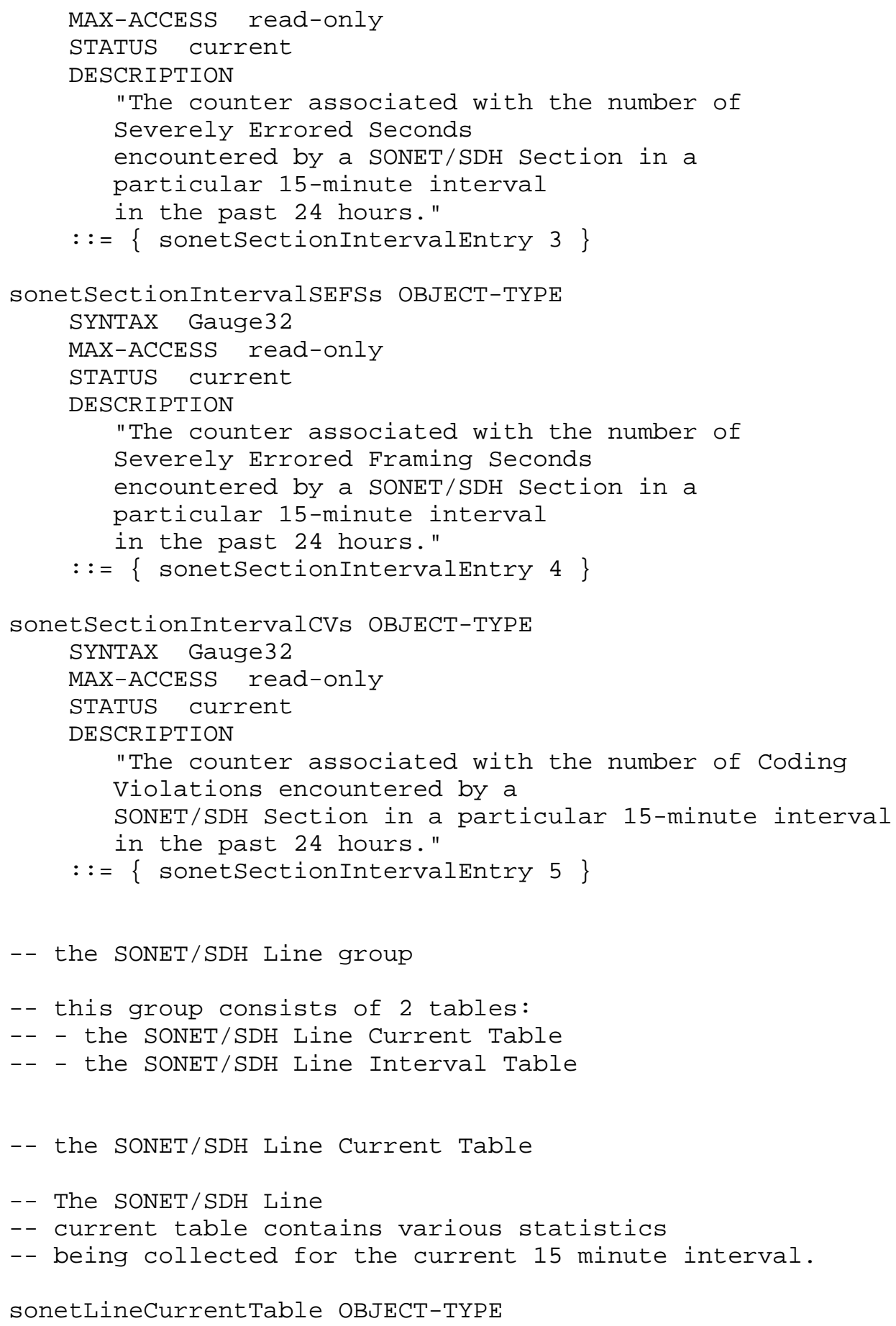




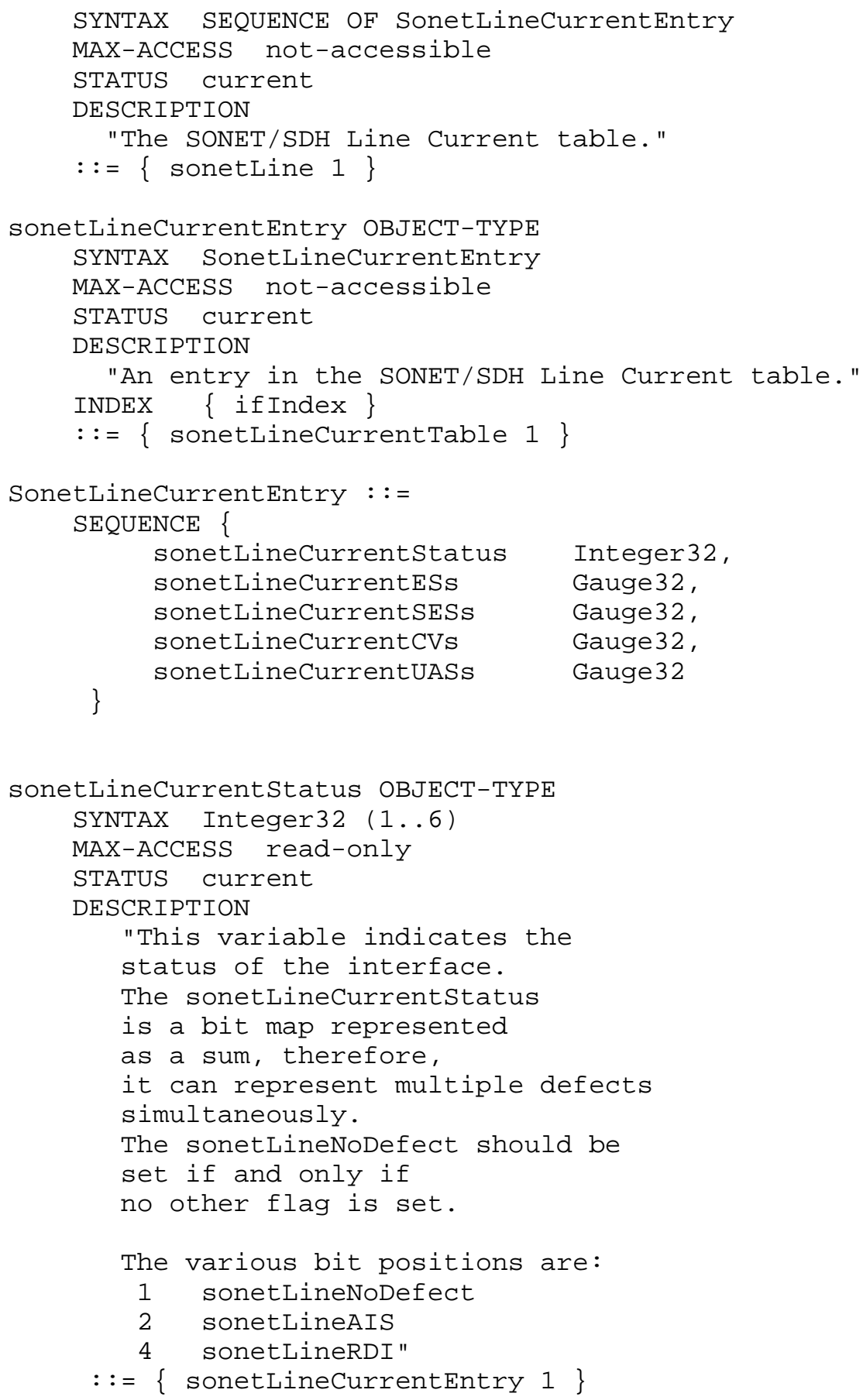

"An entry in the SONET/SDH Line Current table." INDEX \{ifIndex $\}$ 


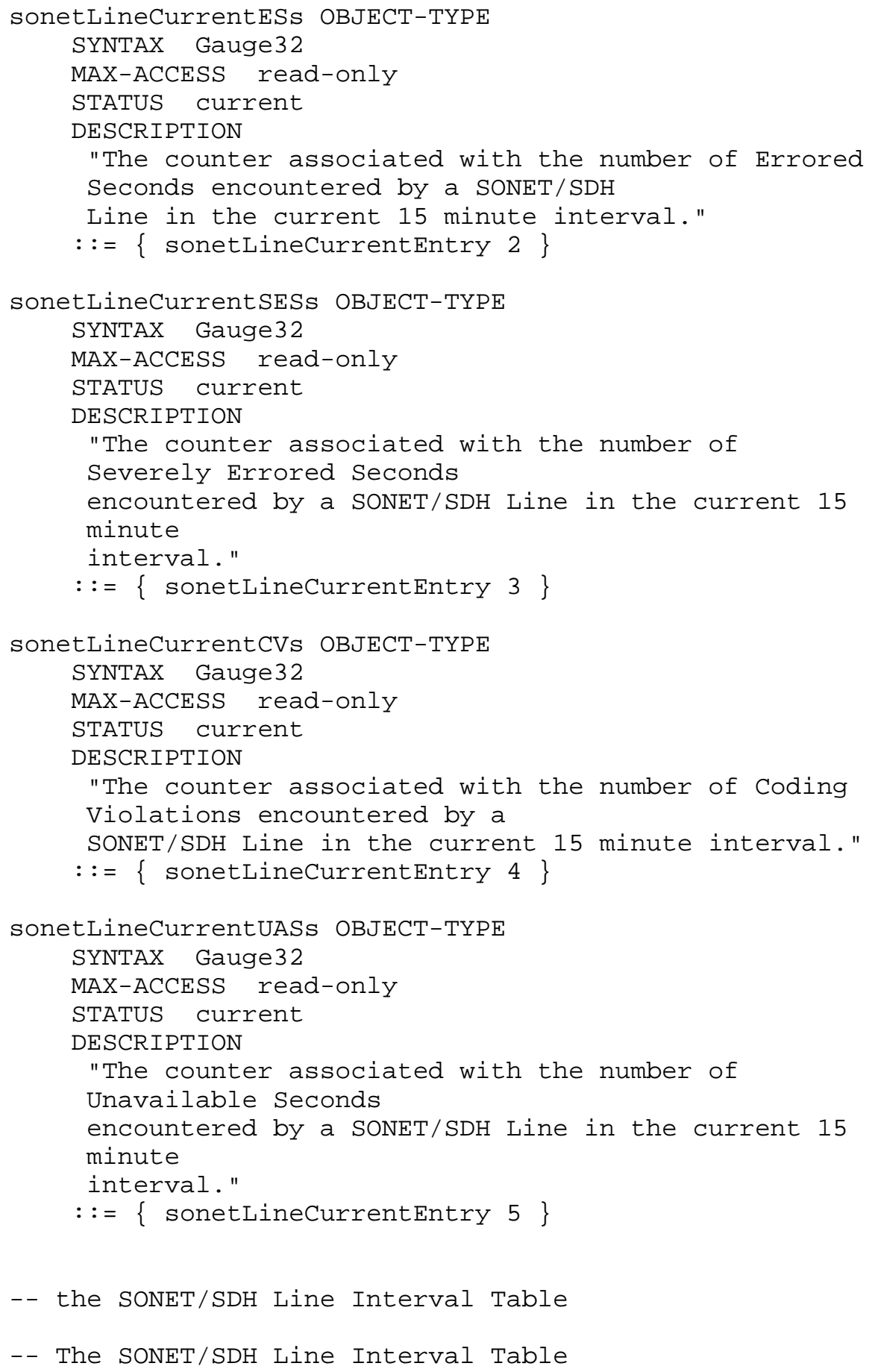




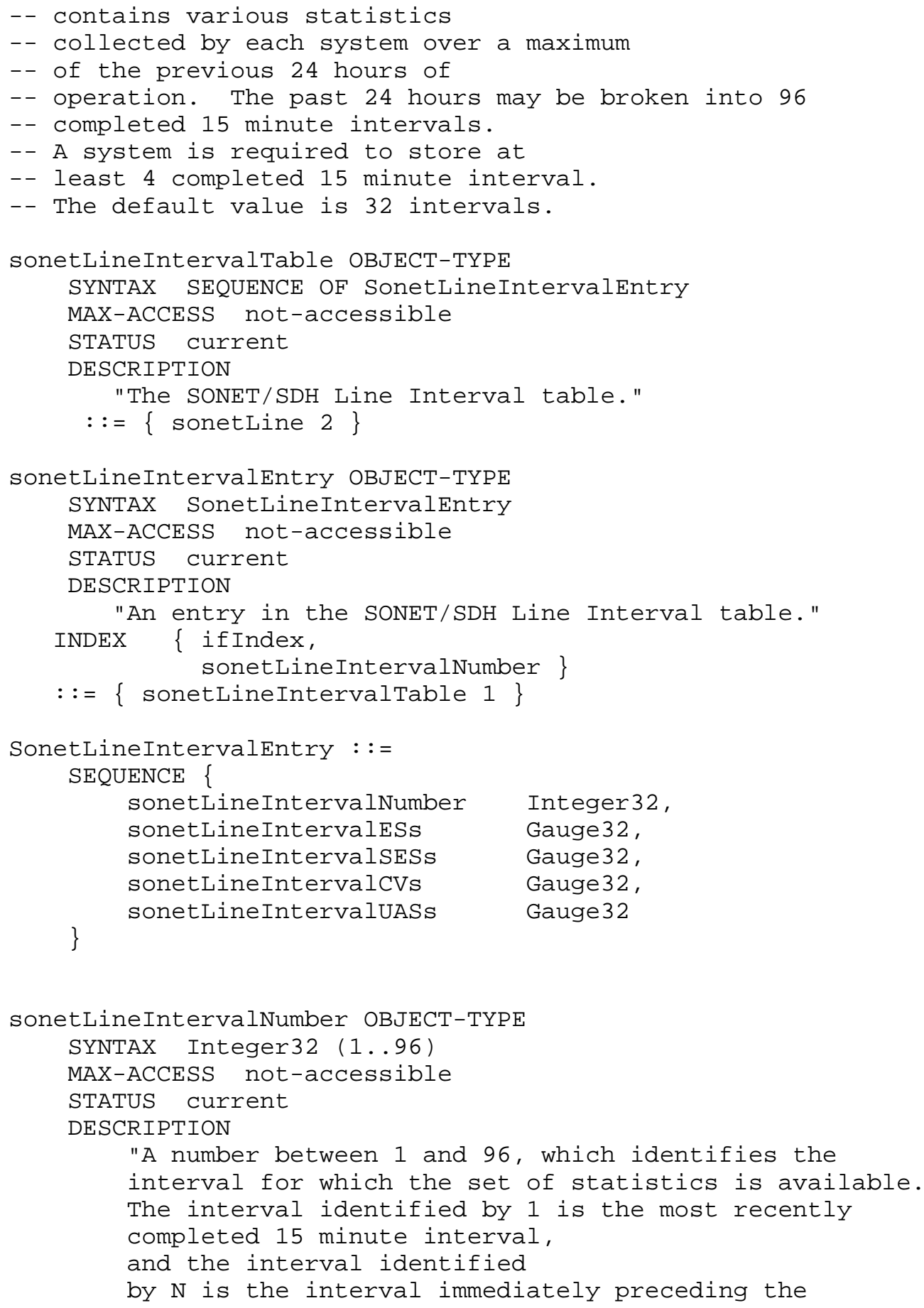




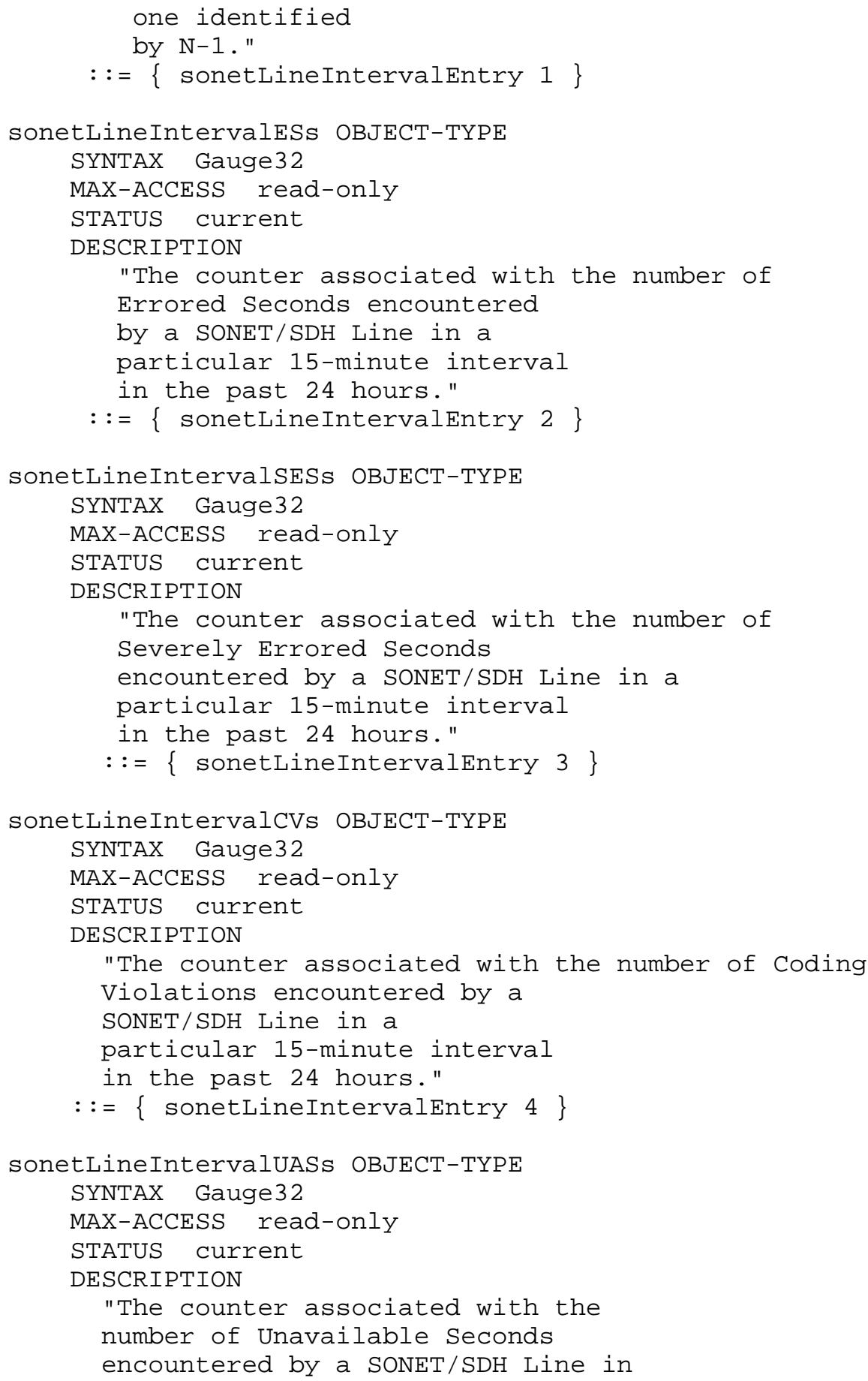




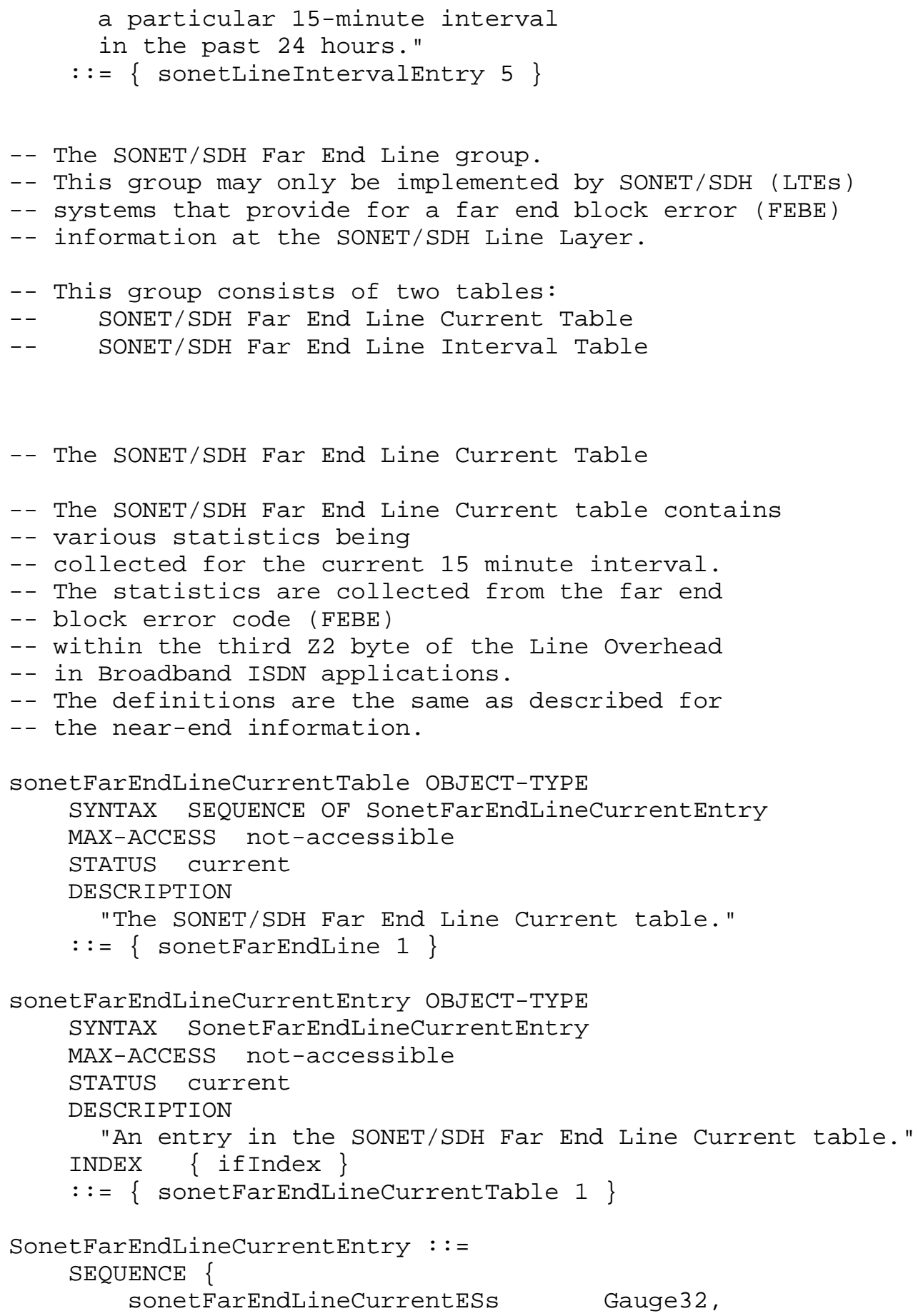




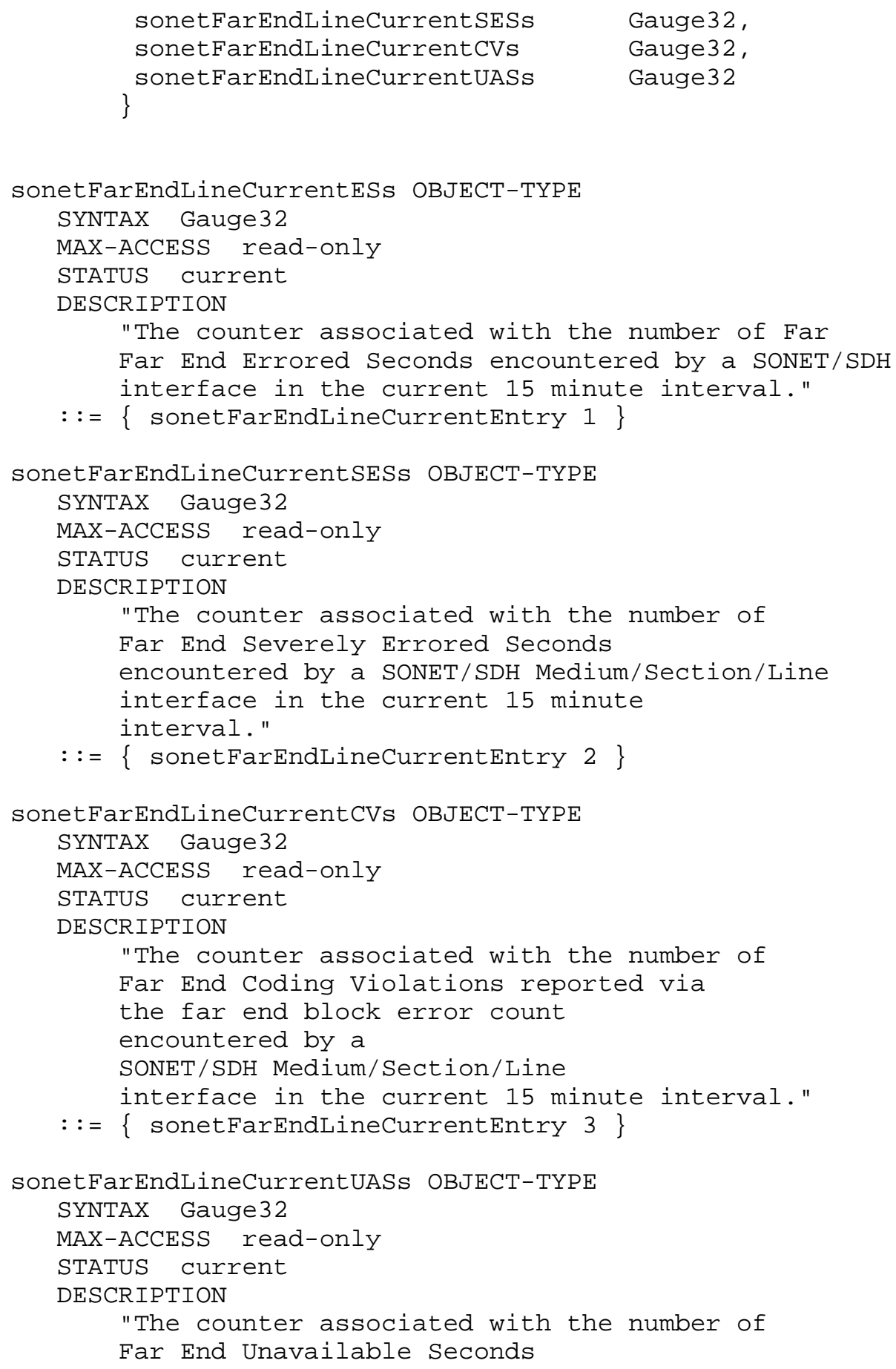




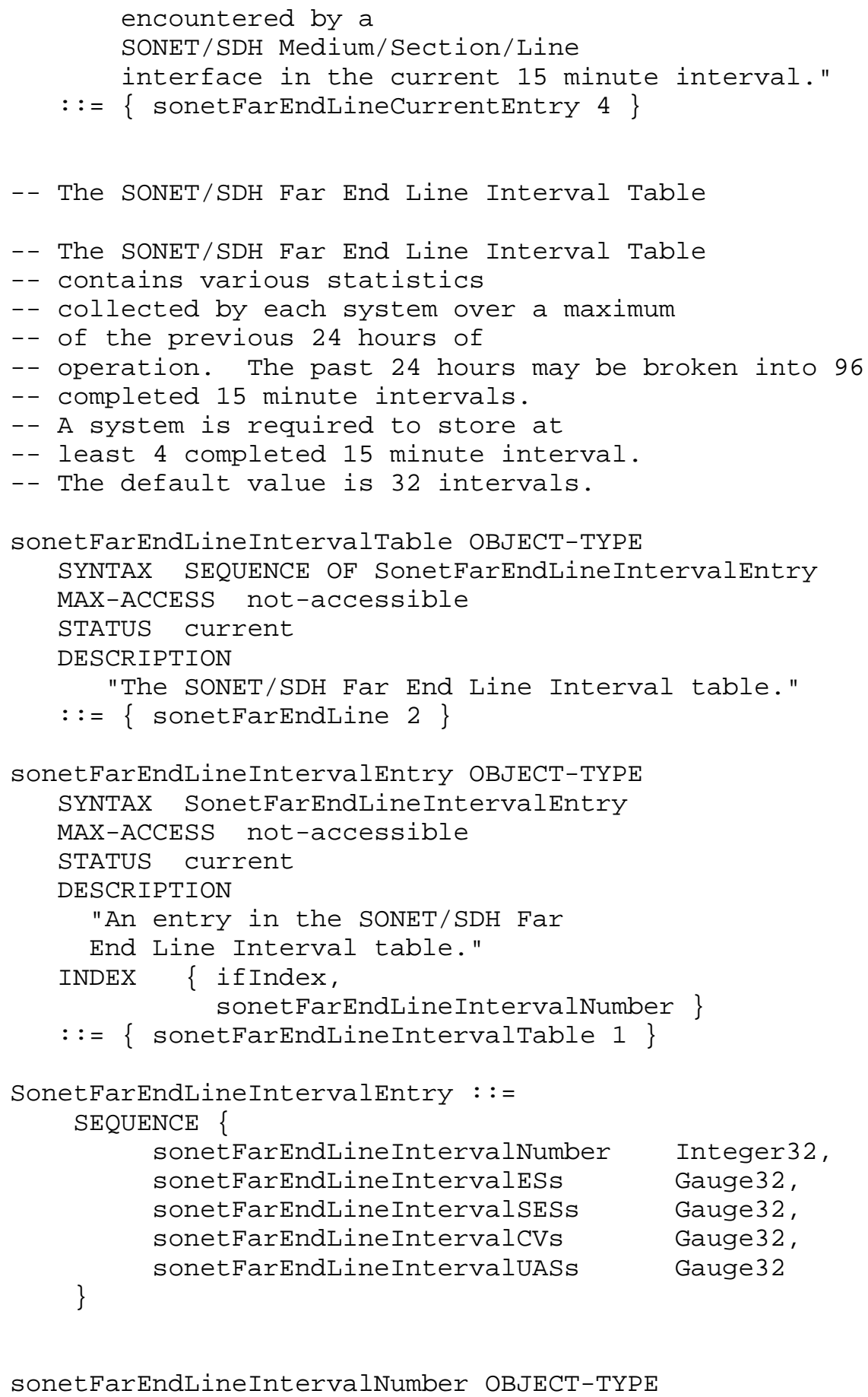




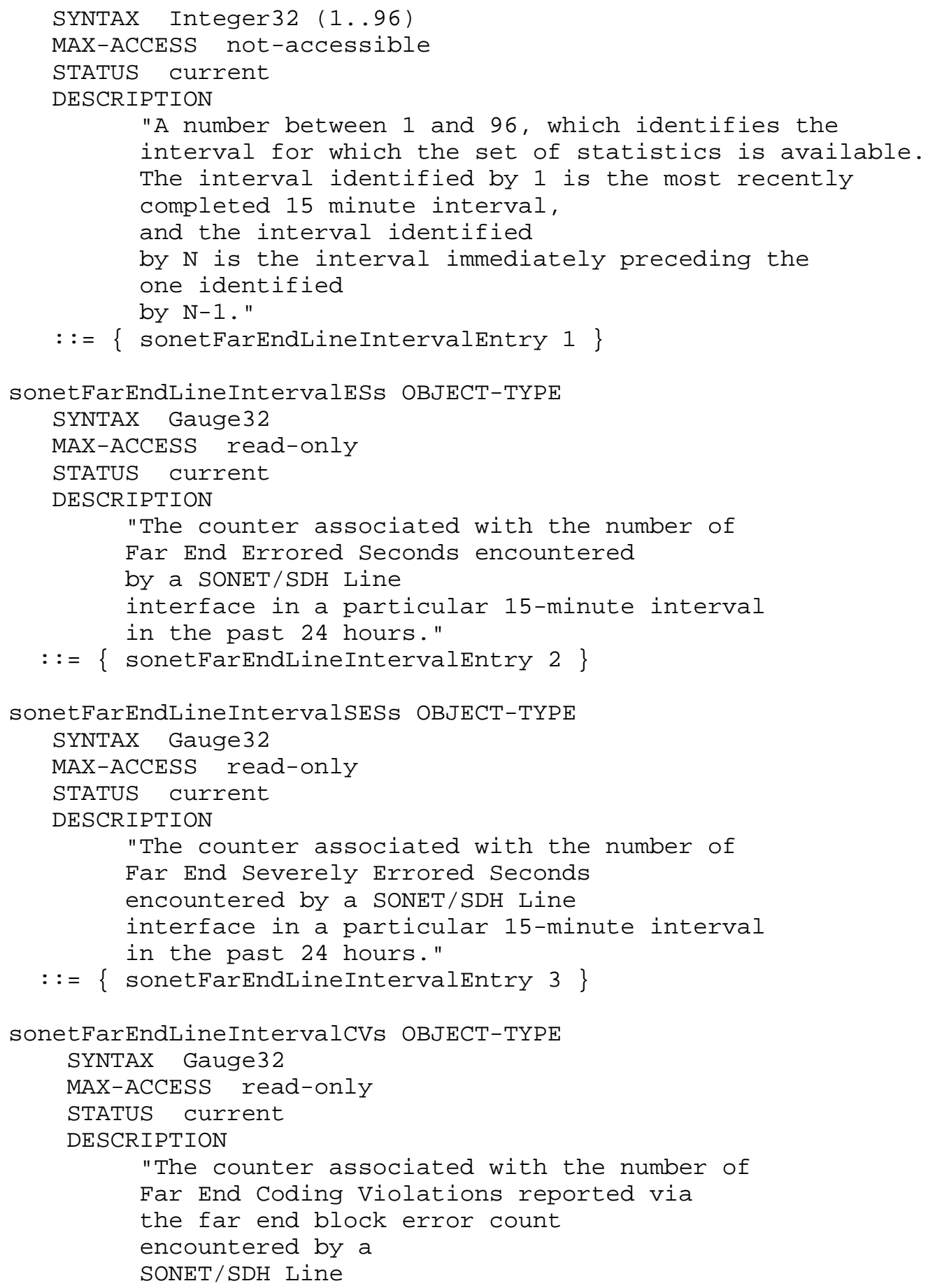




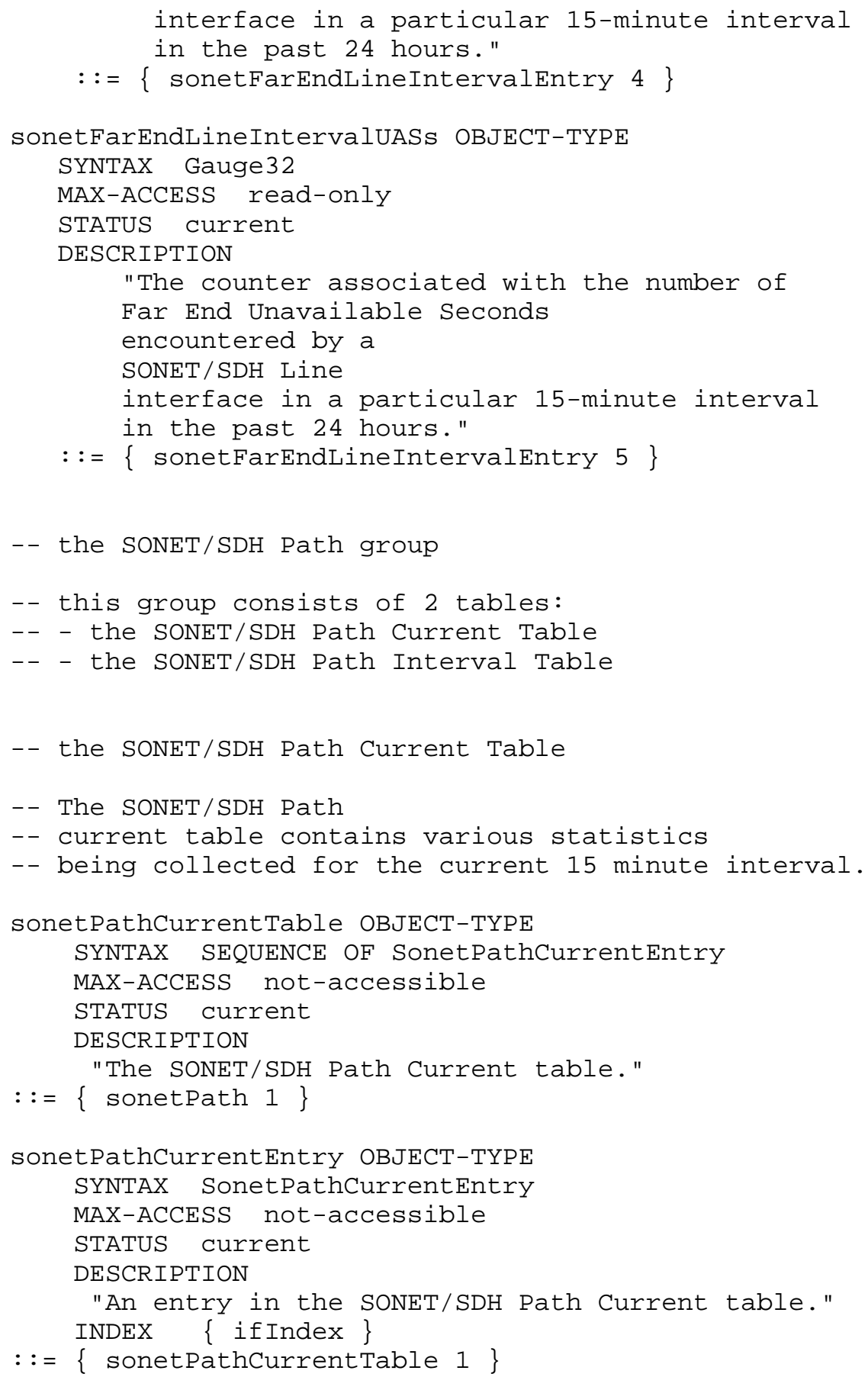




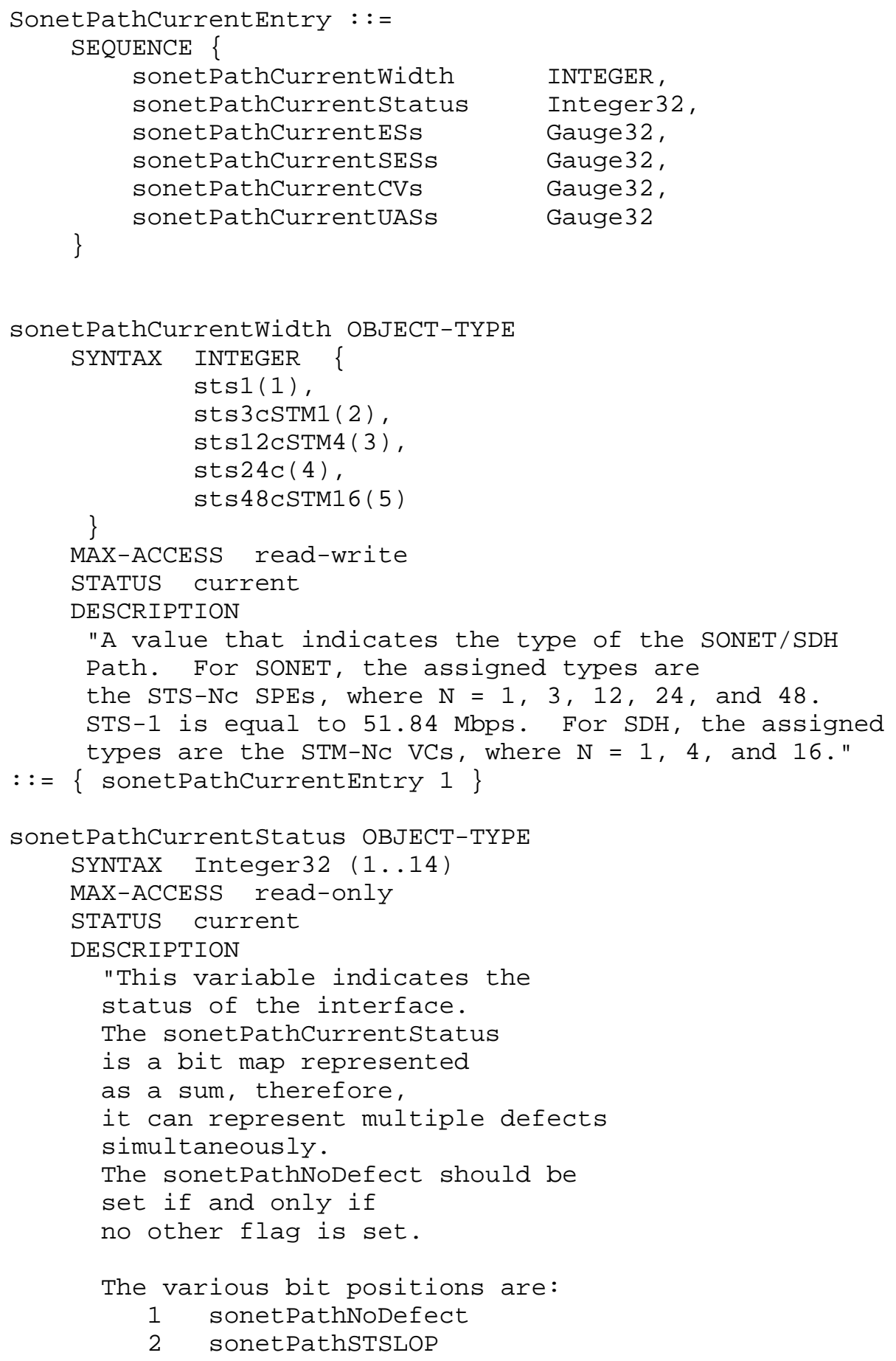




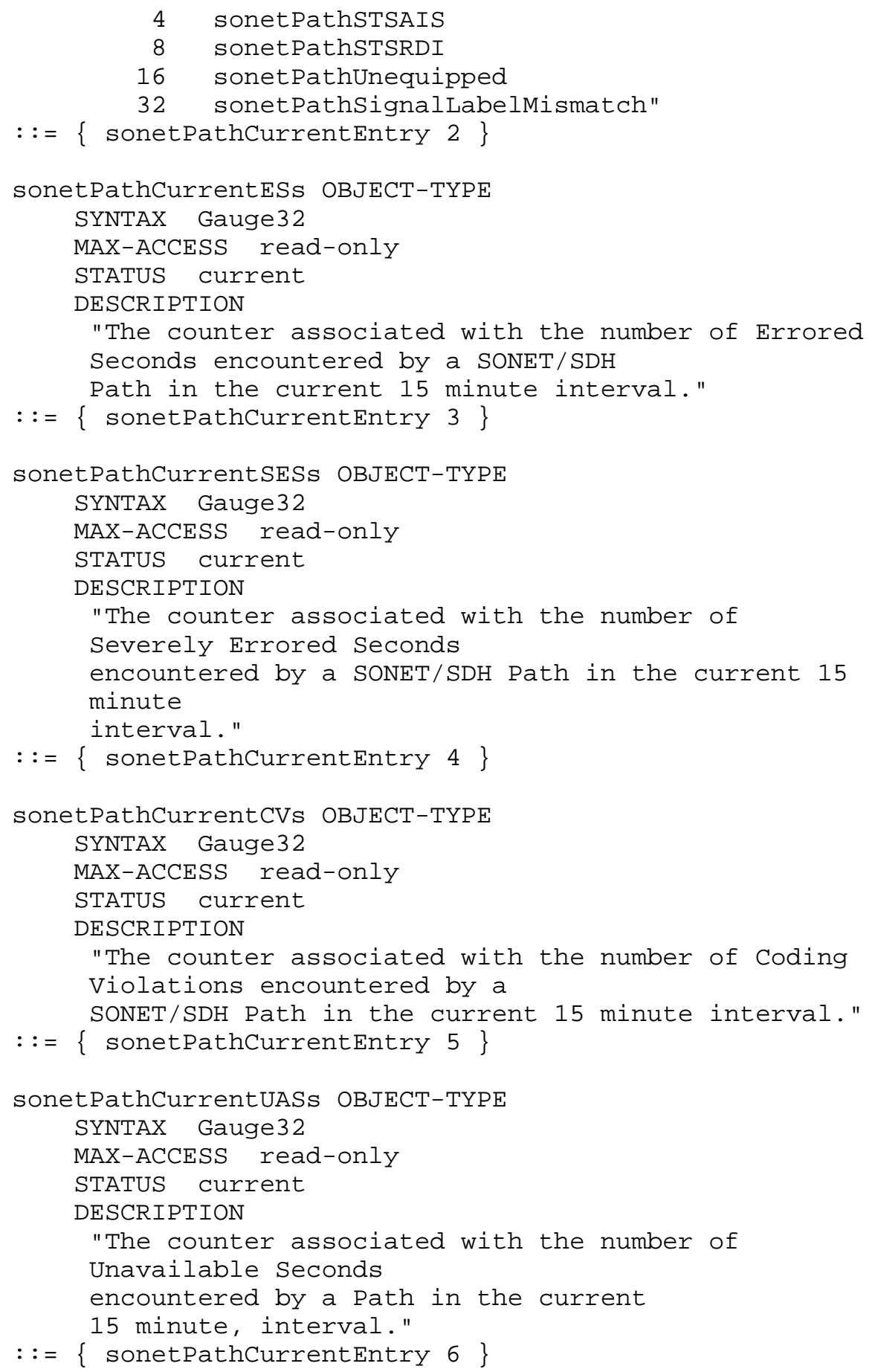


-- the SONET/SDH Path Interval Table

-- The SONET/SDH Path Interval Table

-- contains various statistics

-- collected by each system over a maximum

-- of the previous 24 hours of

-- operation. The past 24 hours may be broken into 96

-- completed 15 minute intervals.

-- A system is required to store at

-- least 4 completed 15 minute interval.

-- The default value is 32 intervals.

sonetPathIntervalTable OBJECT-TYPE

SYNTAX SEQUENCE OF SonetPathIntervalEntry

MAX-ACCESS not-accessible

STATUS current

DESCRIPTION

"The SONET/SDH Path Interval table."

$::=\{$ sonetPath 2$\}$

sonetPathIntervalEntry OBJECT-TYPE

SYNTAX SonetPathIntervalEntry

MAX-ACCESS not-accessible

STATUS current

DESCRIPTION

"An entry in the SONET/SDH Path Interval table." INDEX $\{$ ifIndex, sonetPathIntervalNumber \}

$::=\{$ sonetPathIntervalTable 1$\}$

SonetPathIntervalEntry : := SEQUENCE \{ sonetPathIntervalNumber Integer32, sonetPathIntervalESs Gauge32, sonetPathIntervalSESs Gauge32, sonetPathIntervalCVs Gauge32, sonetPathIntervalUASs Gauge32 \}

sonetPathIntervalNumber OBJECT-TYPE

SYNTAX Integer32 (1..96)

MAX-ACCESS not-accessible

STATUS current

DESCRIPTION

"A number between 1 and 96, which identifies the

interval for which the set of statistics is available. 


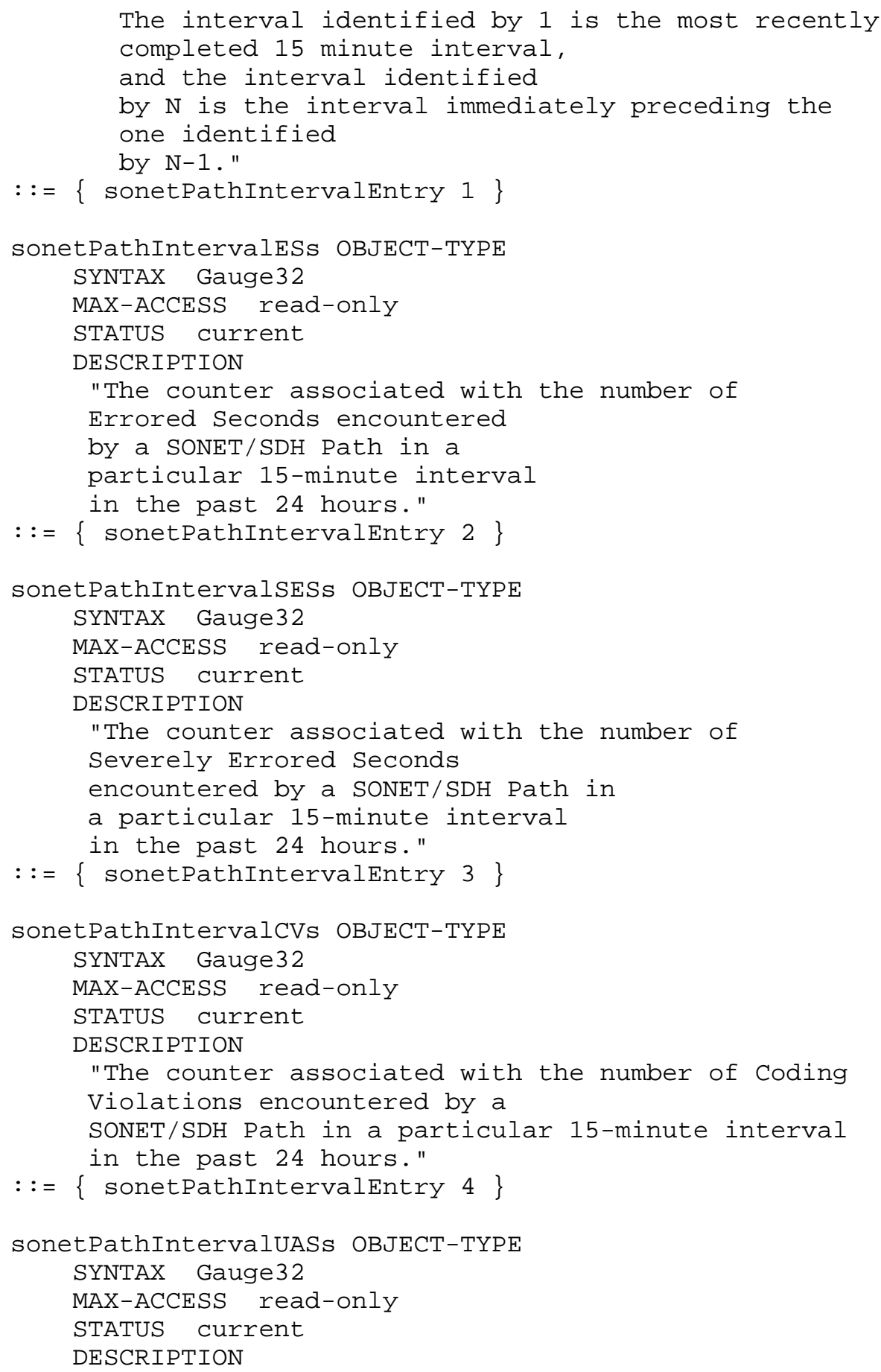




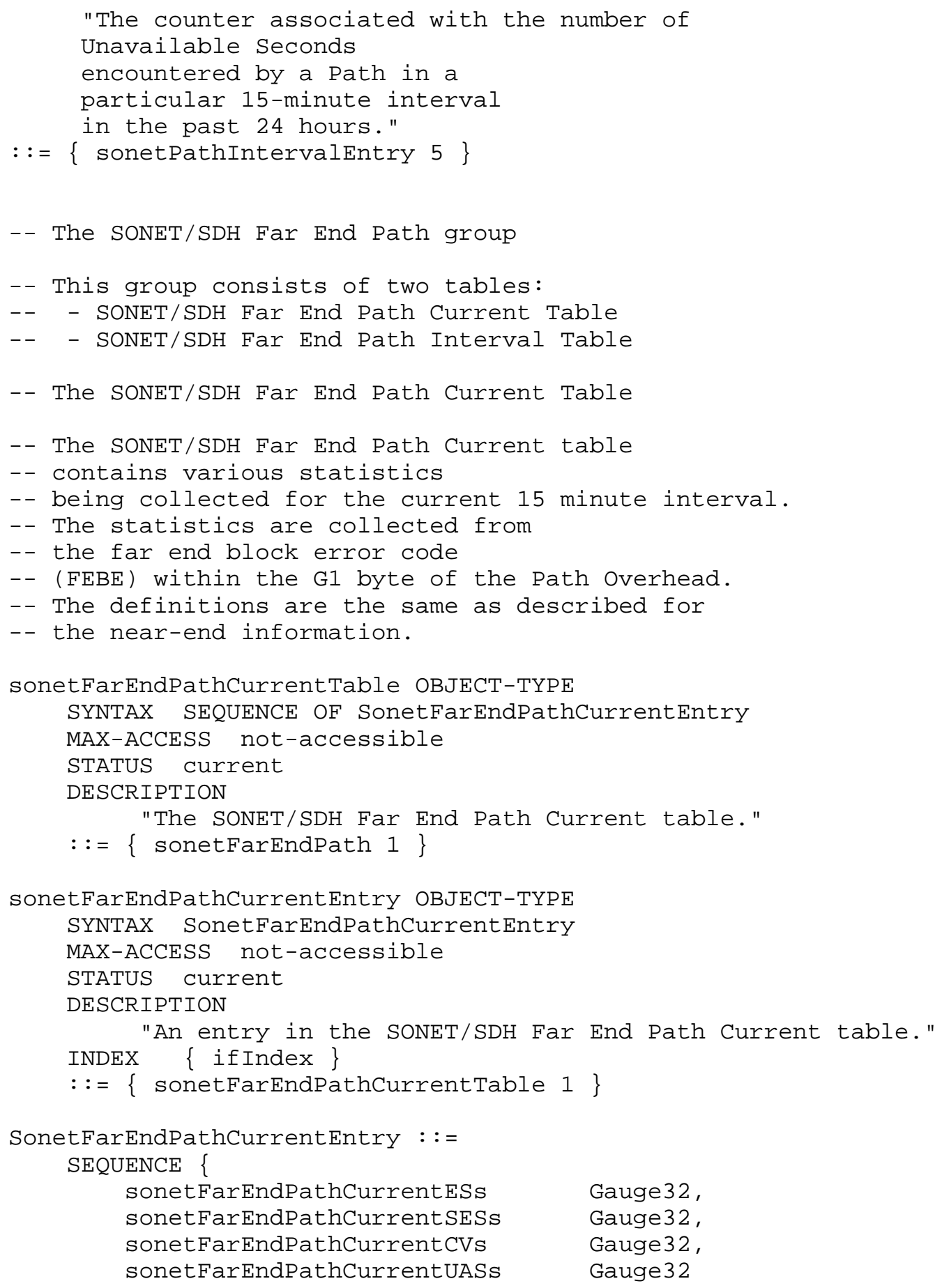




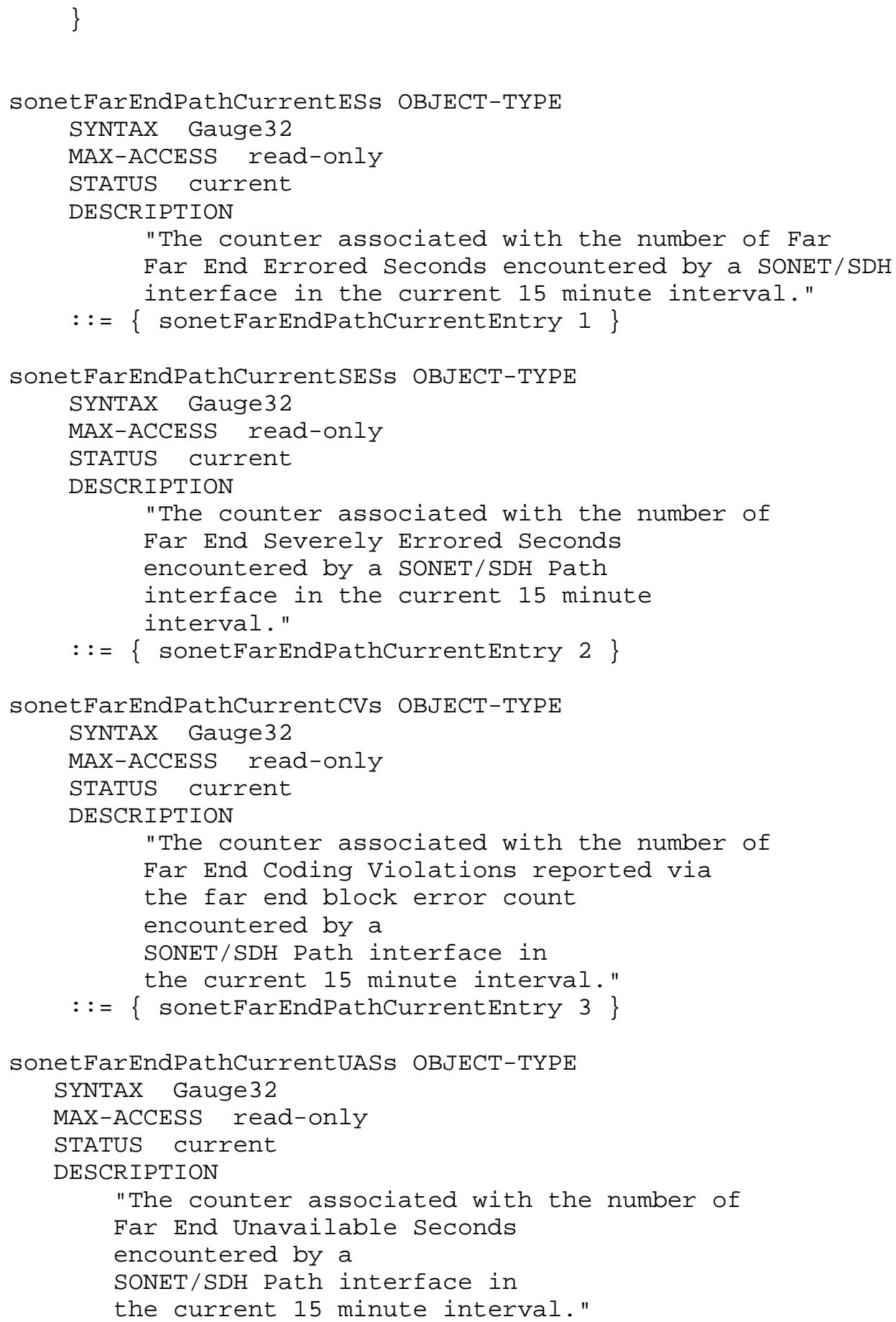




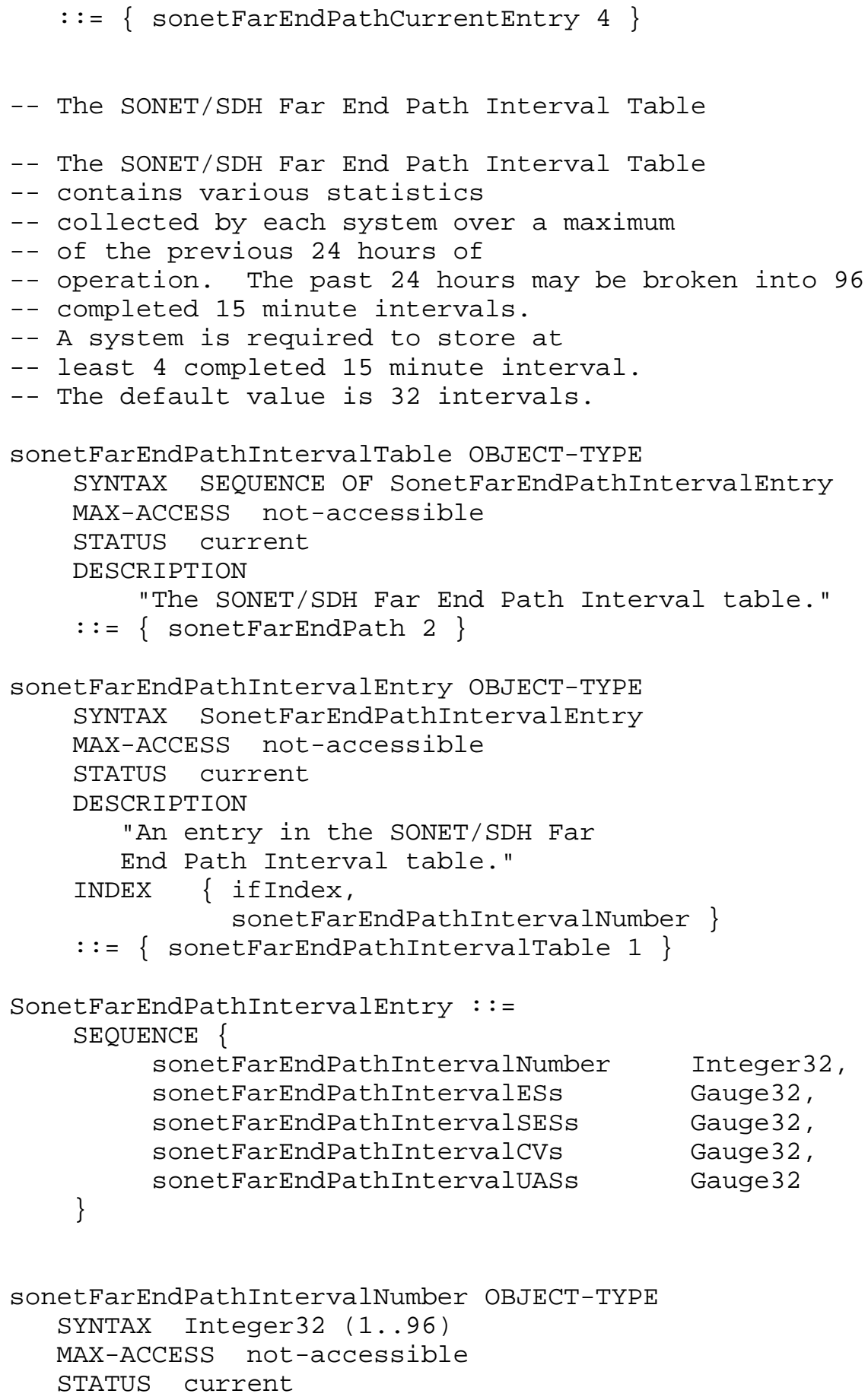




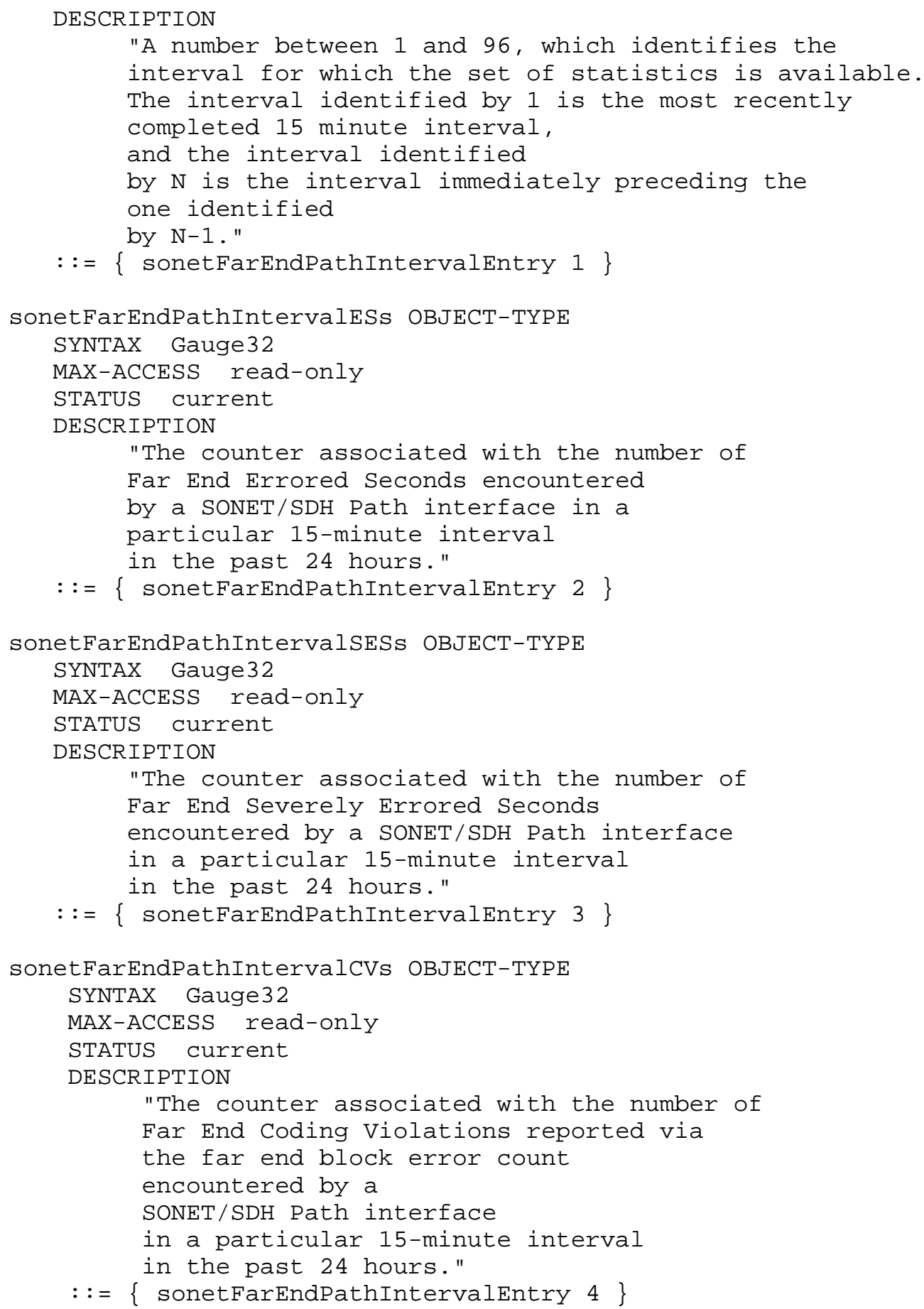




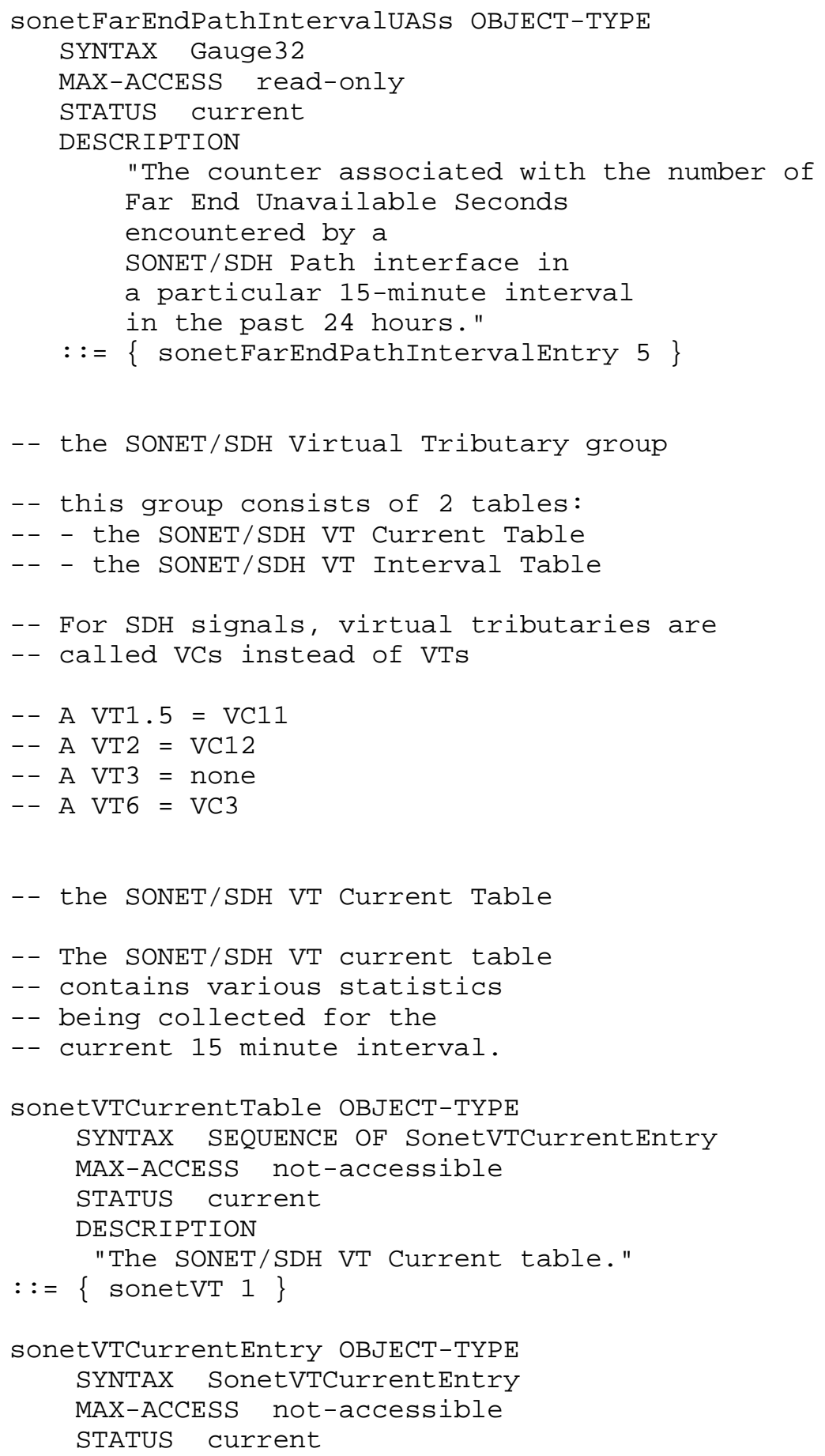




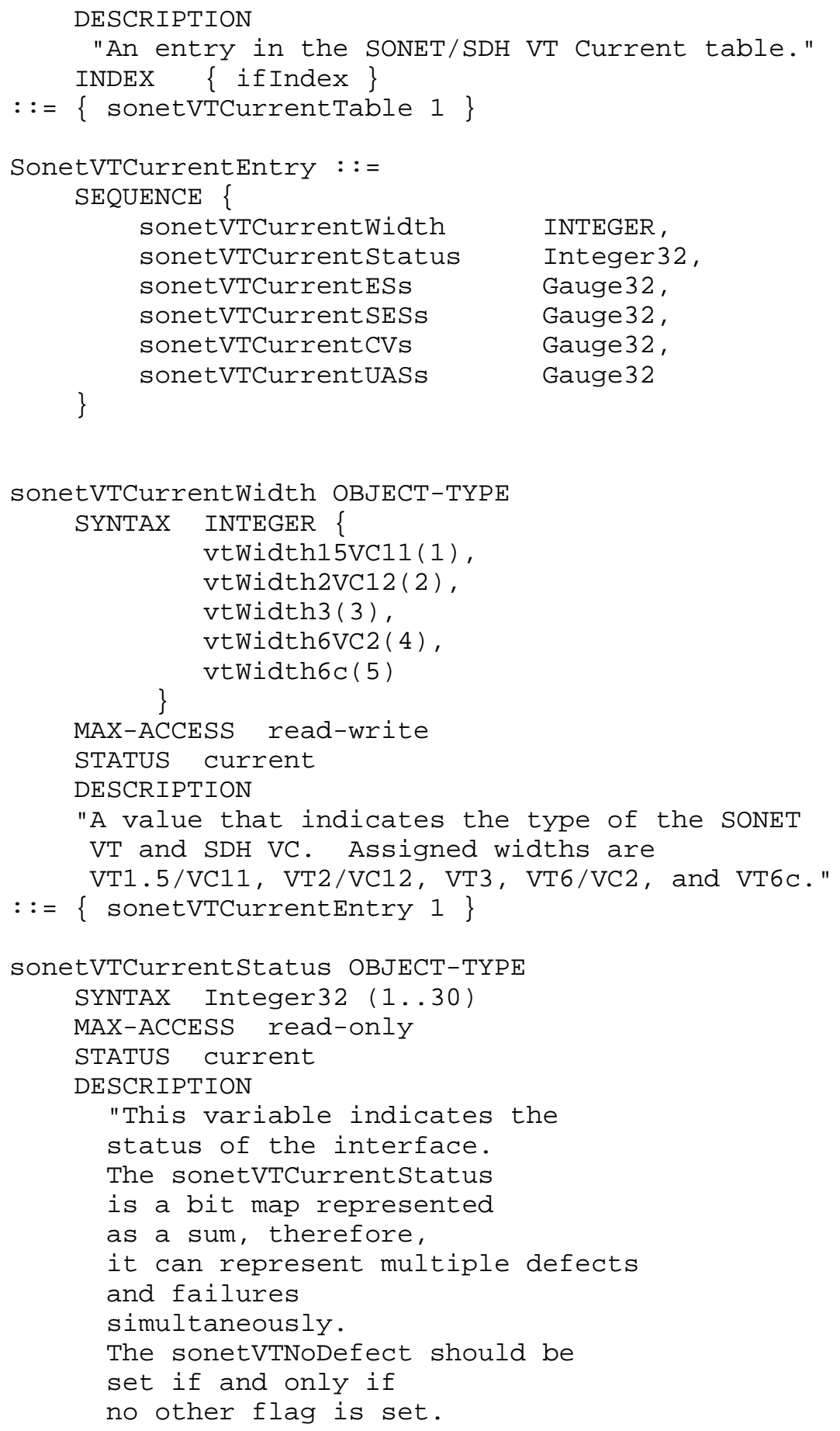




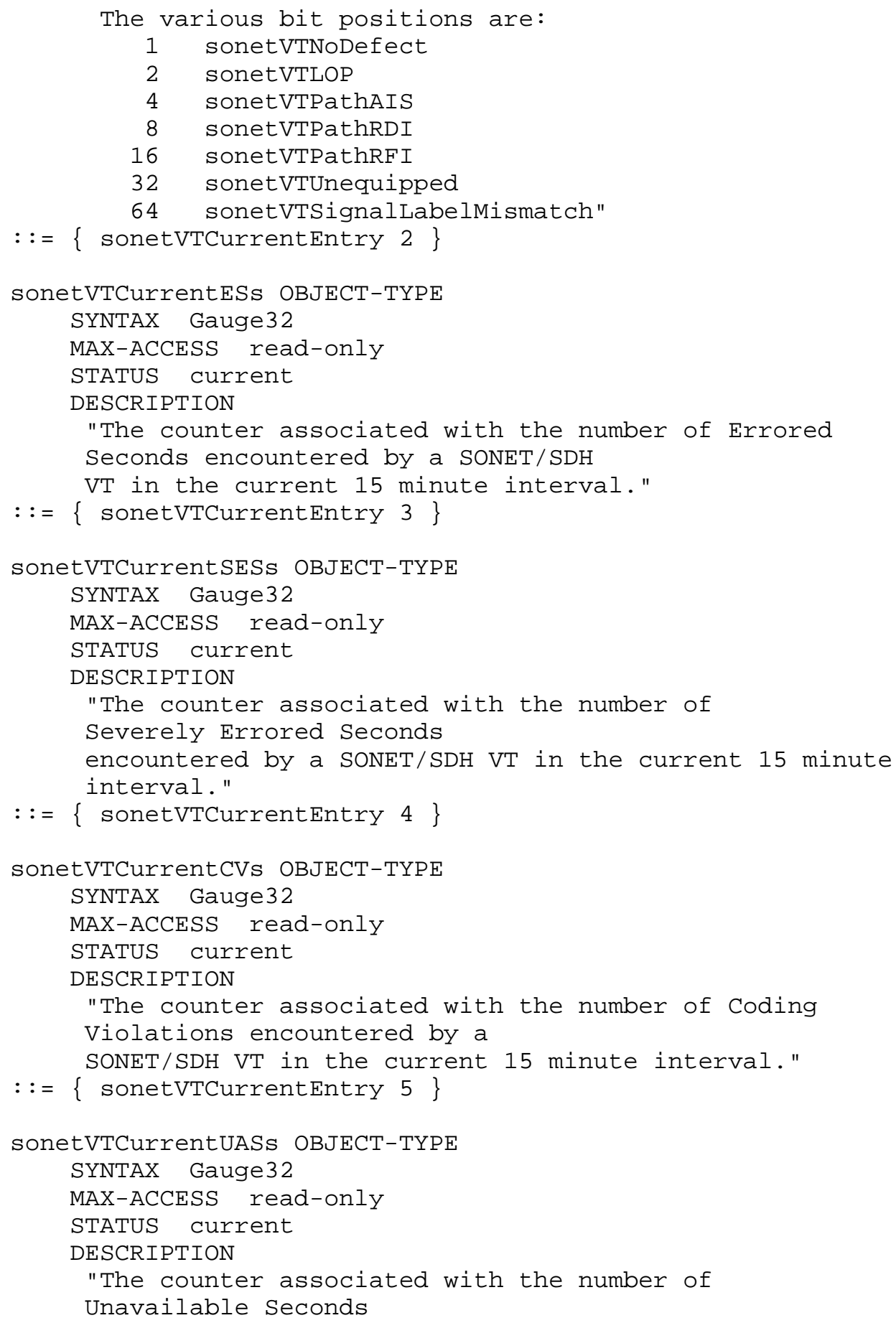




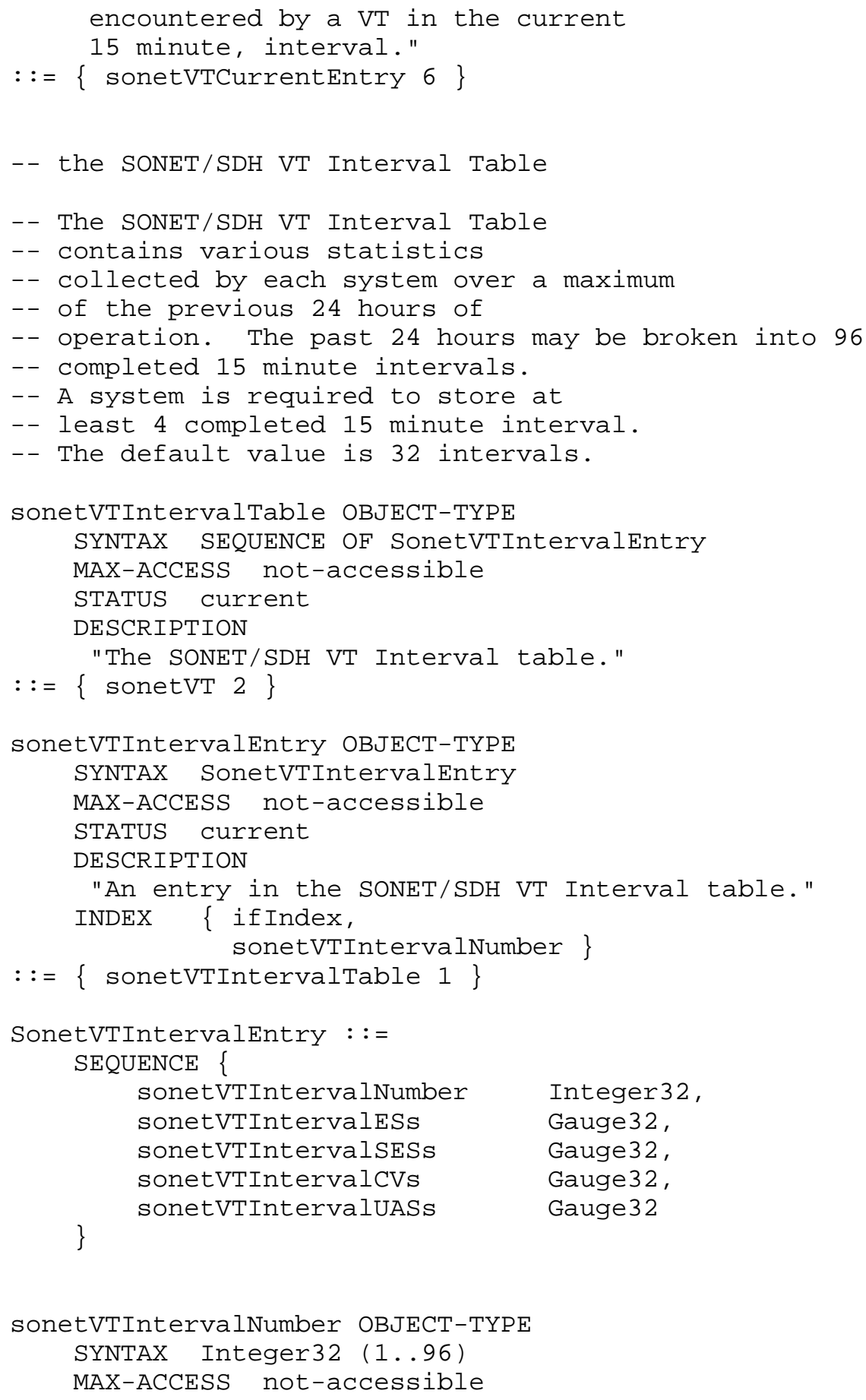




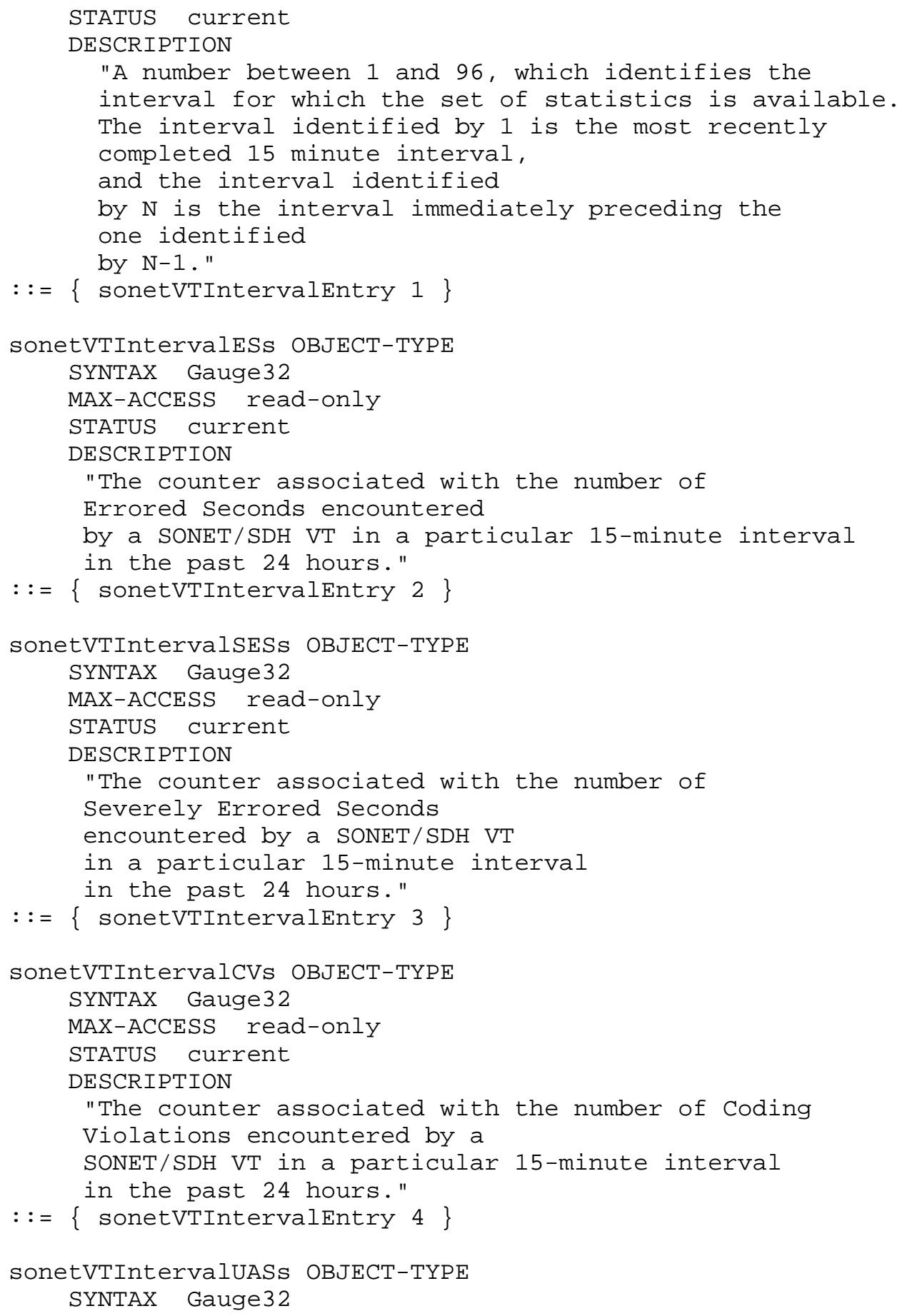




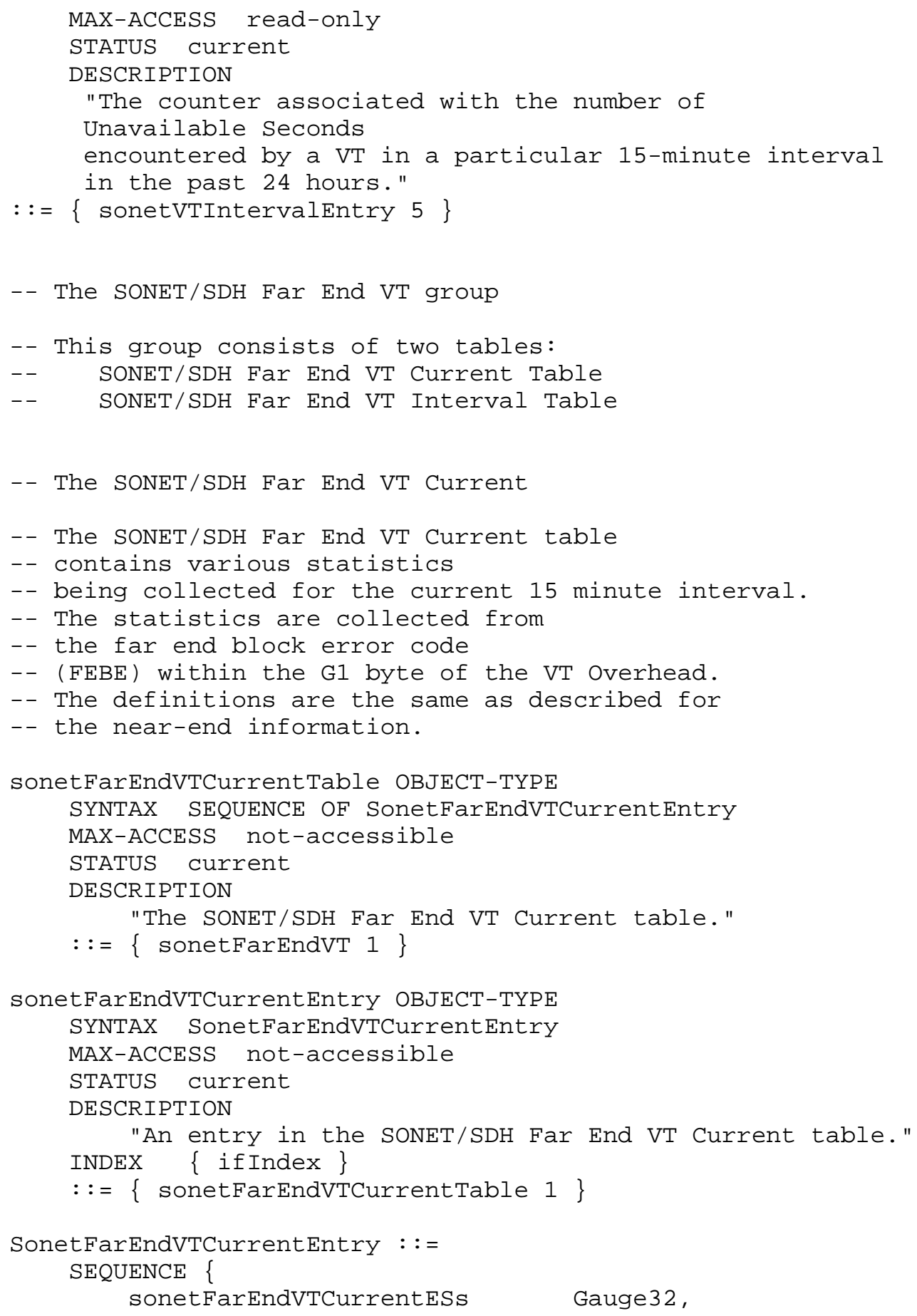




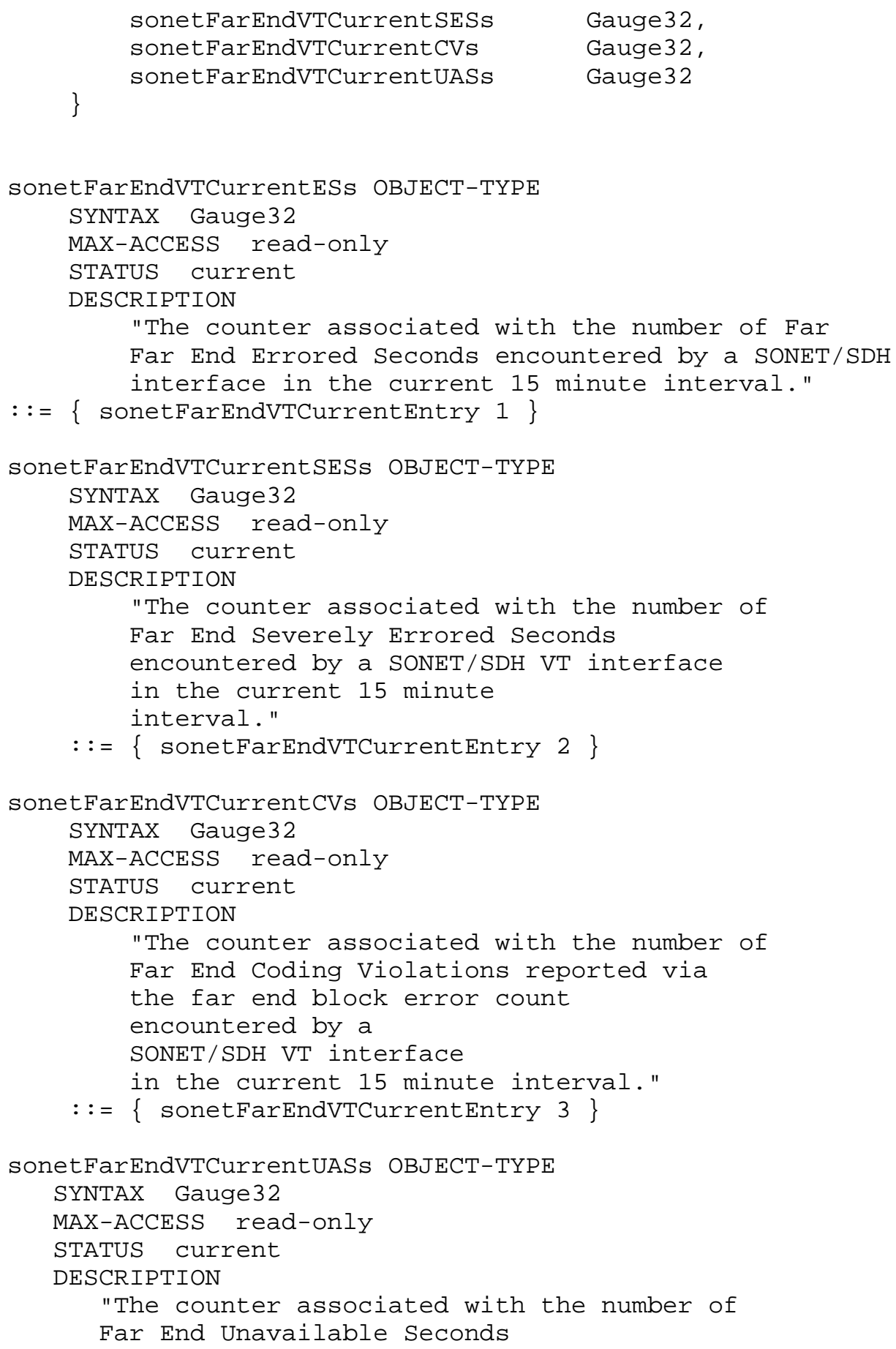




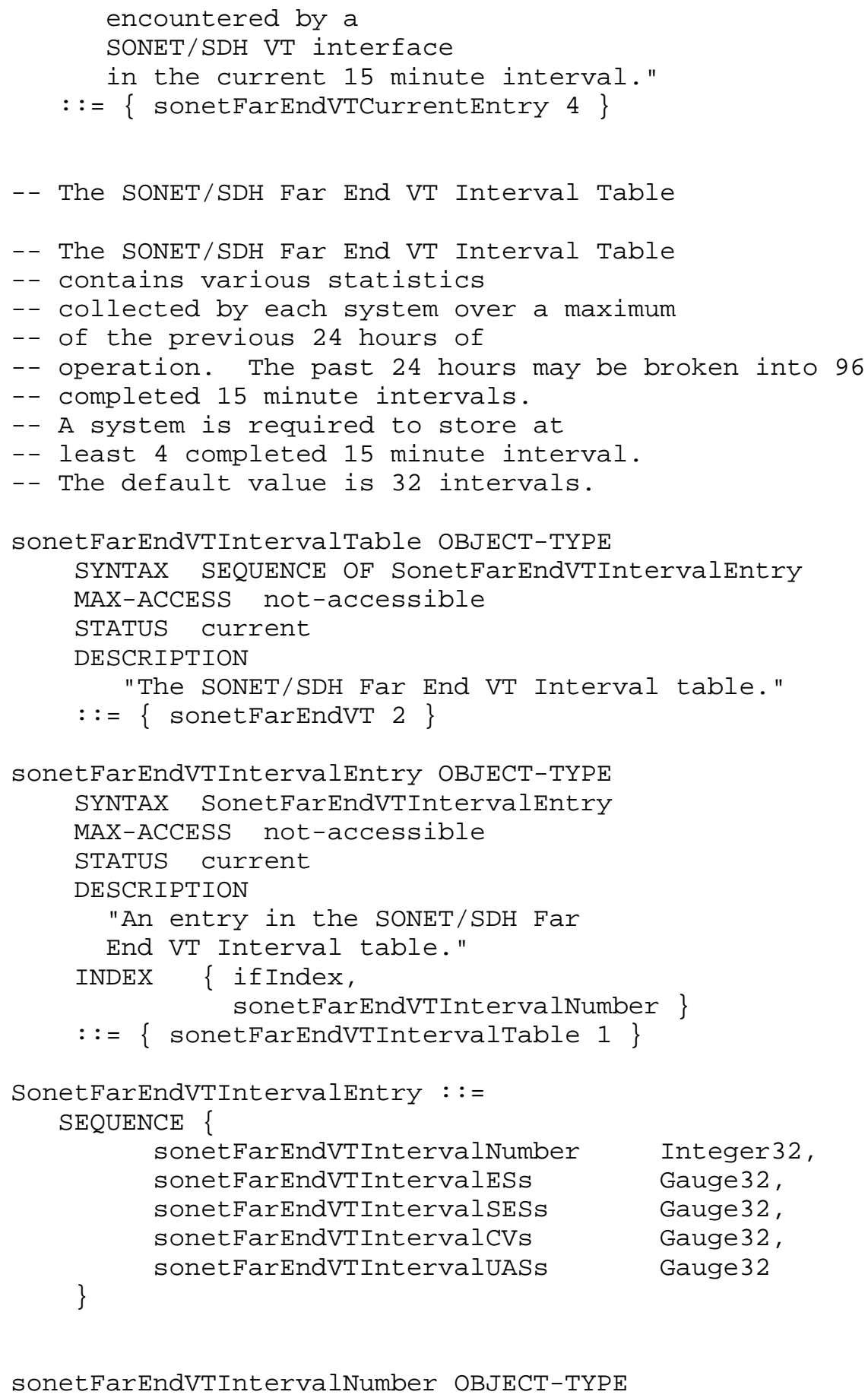




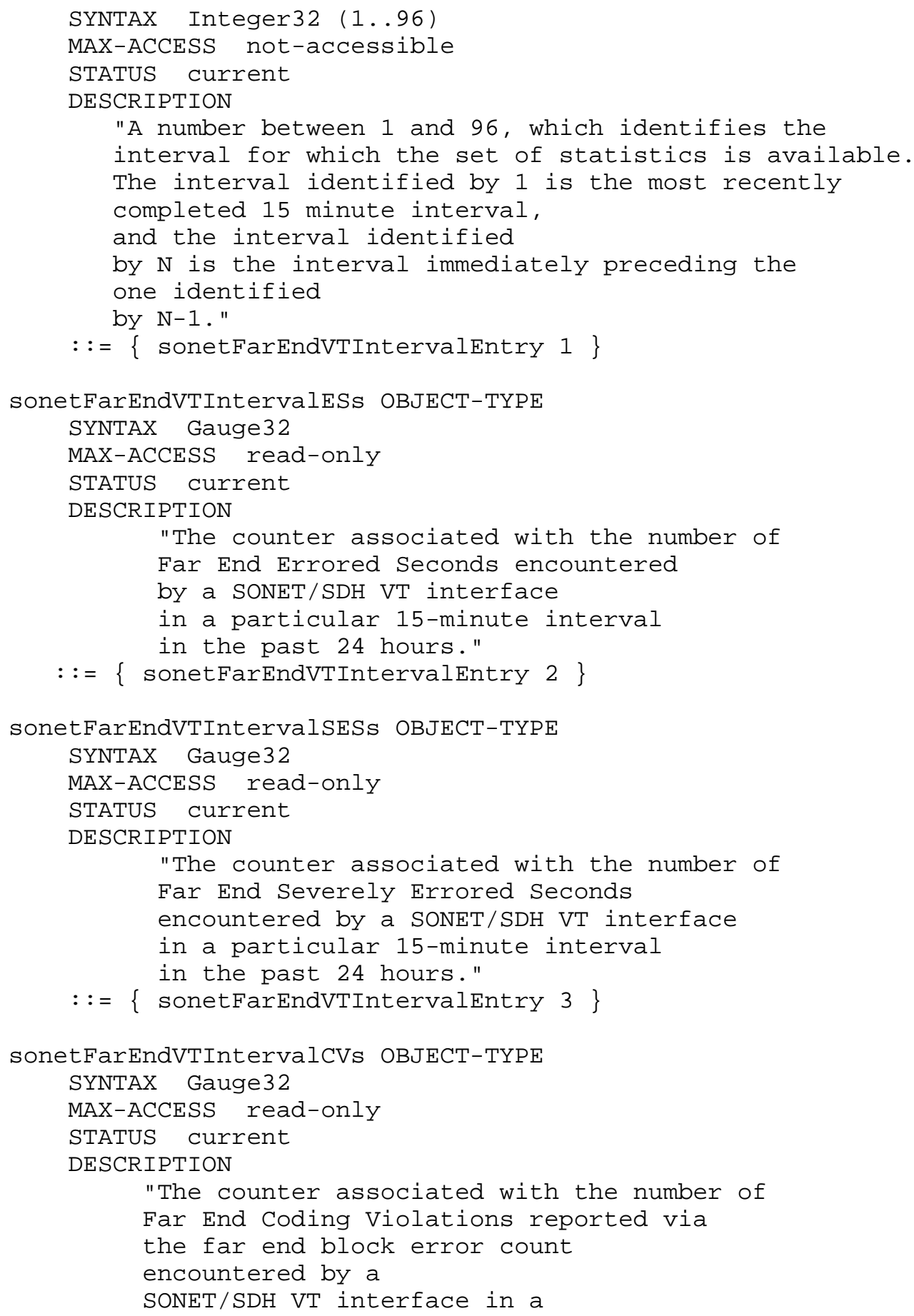




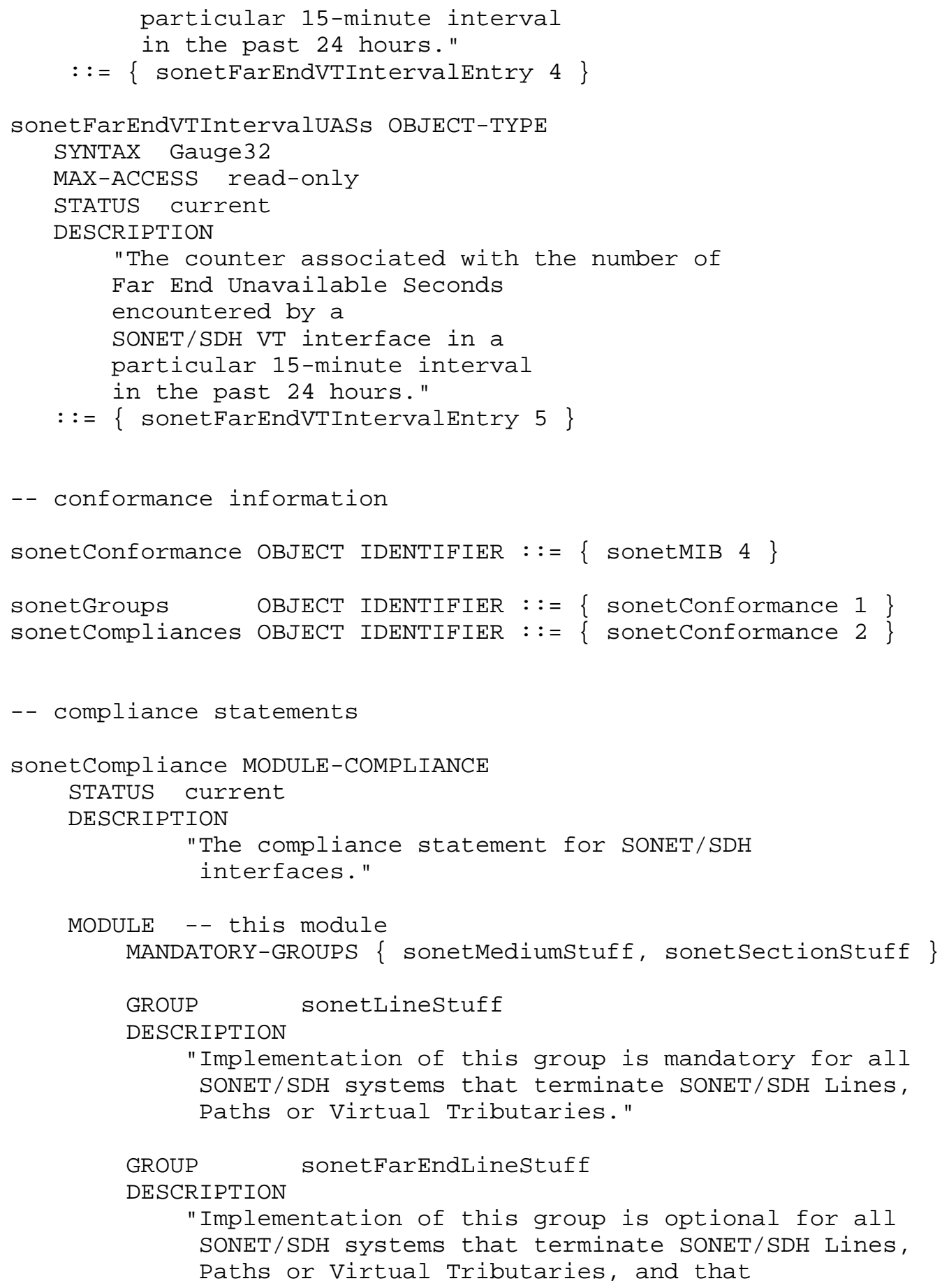




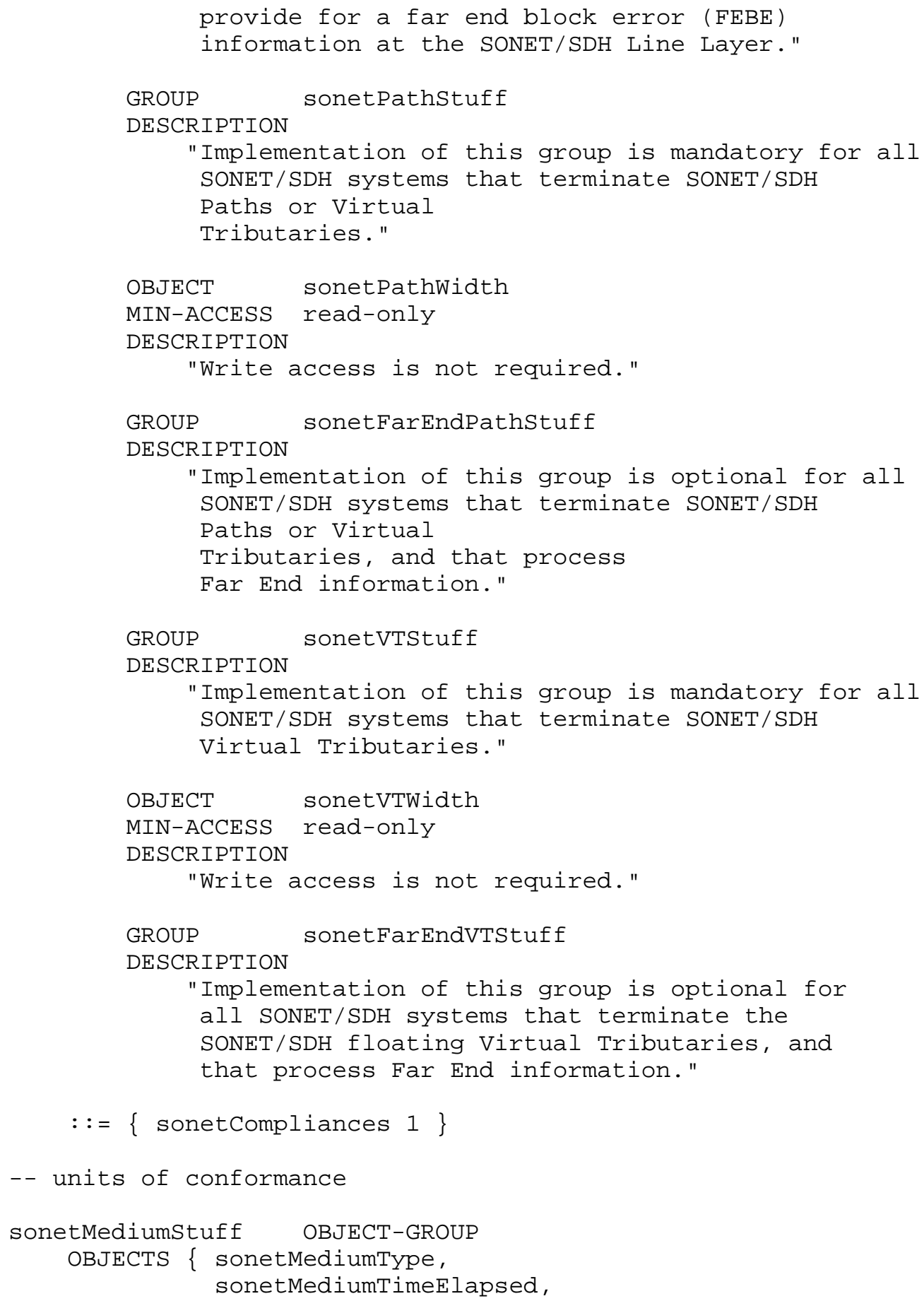




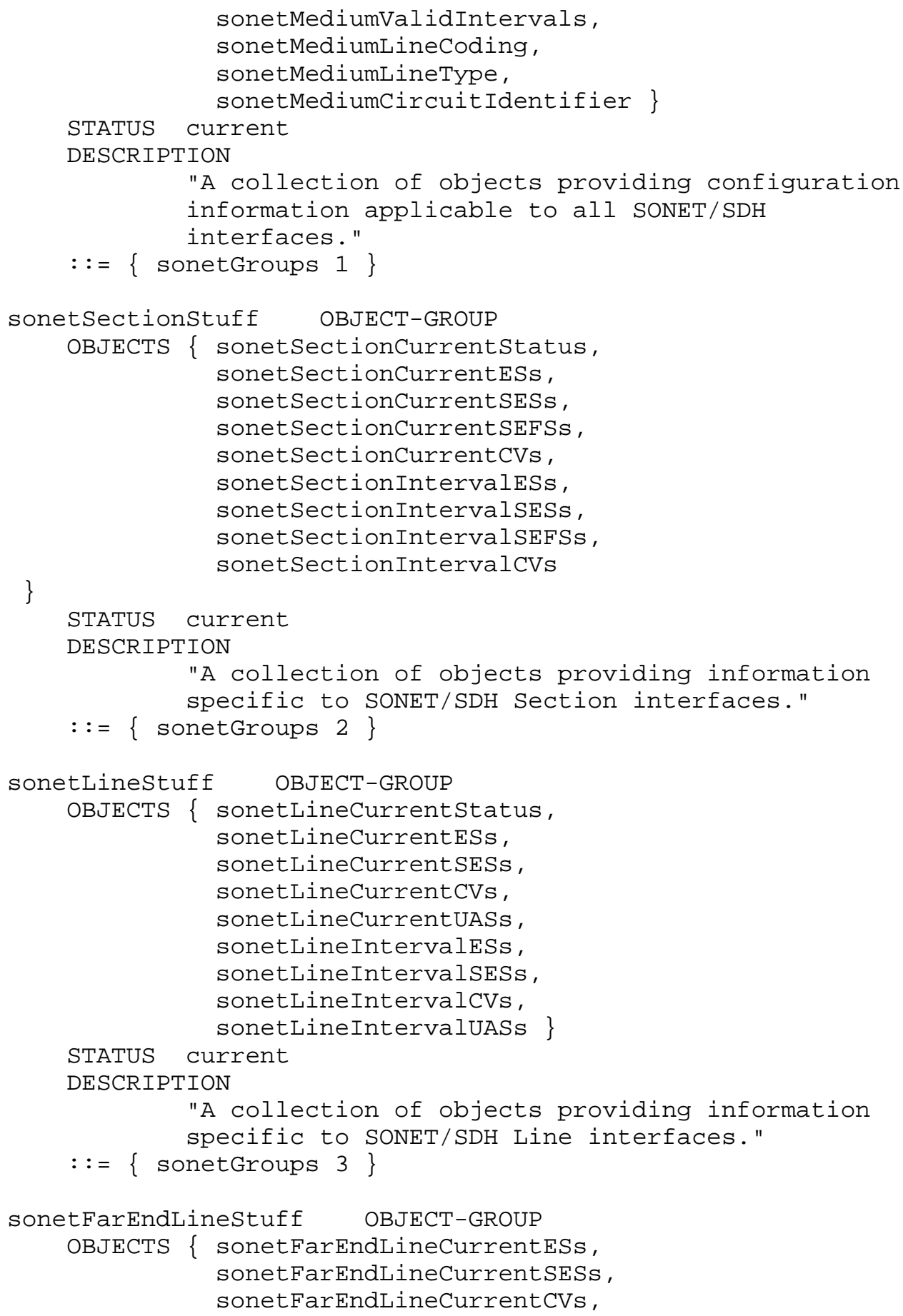




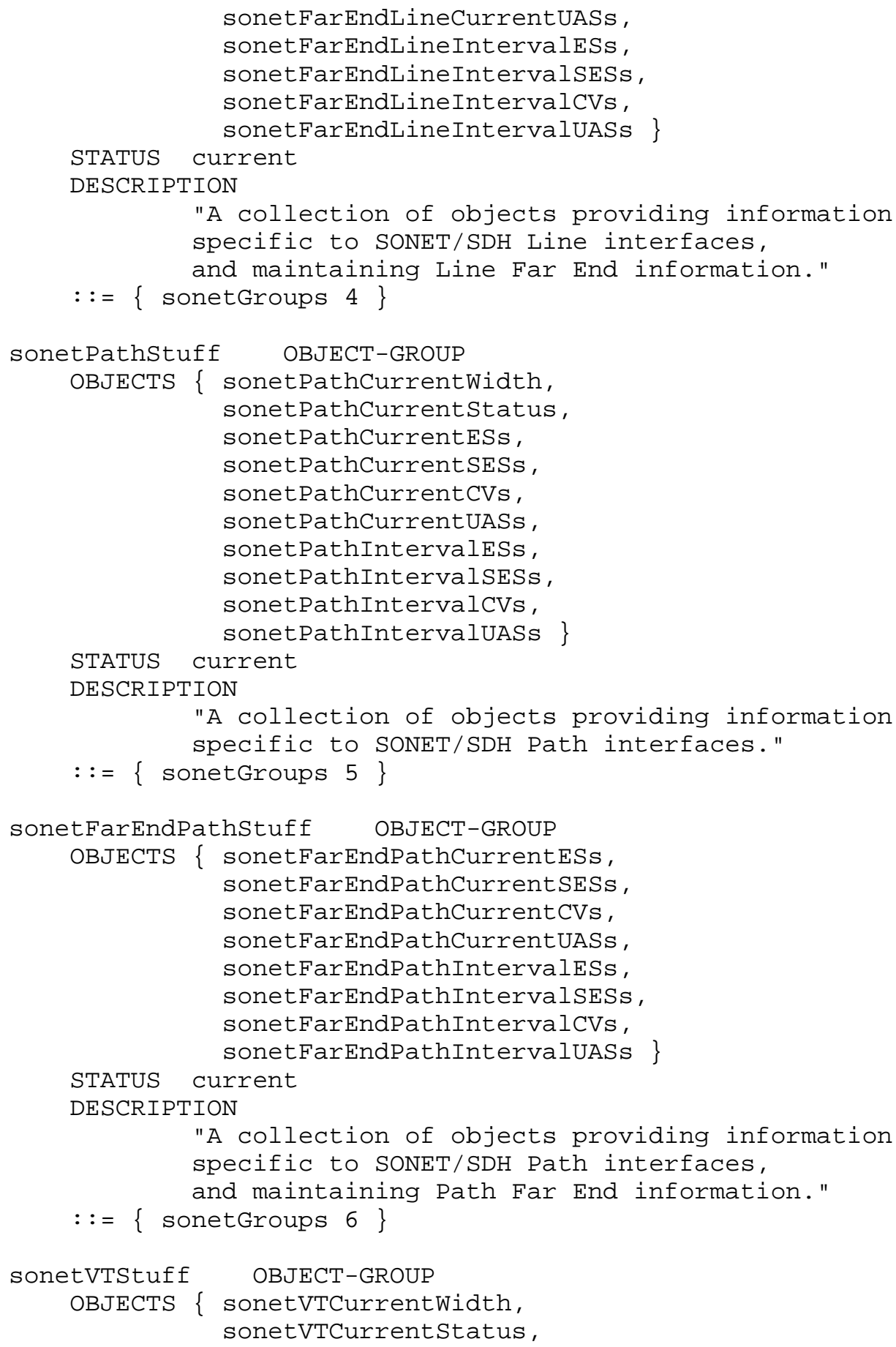

"A collection of objects providing information specific to SONET/SDH Line interfaces, and maintaining Line Far End information."

"A collection of objects providing information specific to SONET/SDH Path interfaces."

"A collection of objects providing information specific to SONET/SDH Path interfaces, and maintaining Path Far End information." $::=\{$ sonetGroups 6$\}$

sonetVTStuff OBJECT-GROUP

OBJECTS \{ sonetVTCurrentWidth, sonetVTCurrentstatus, 


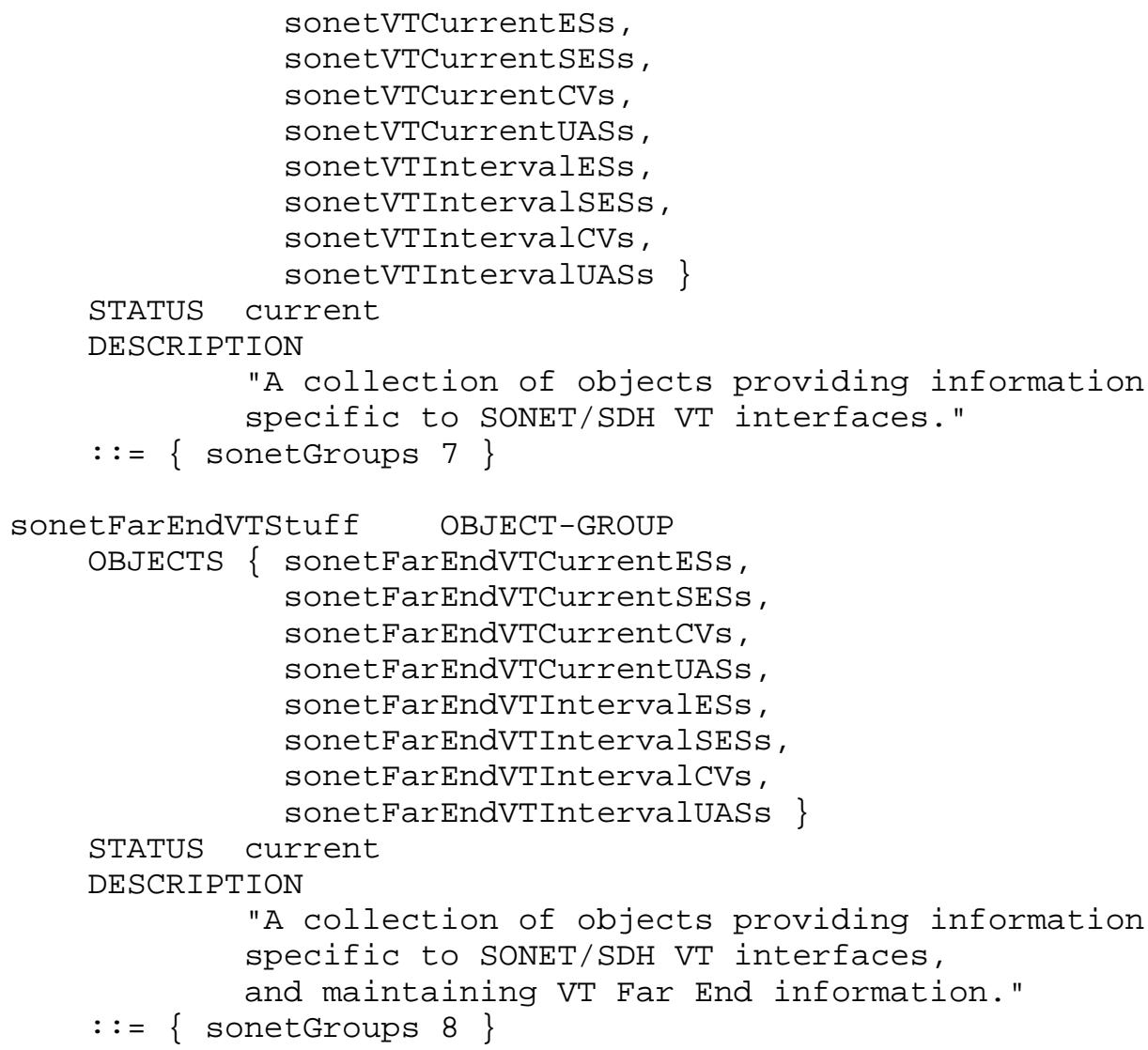

"A collection of objects providing information specific to SONET/SDH VT interfaces, and maintaining VT Far End information." $::=\{$ sonetGroups 8$\}$

END

5. Acknowledgments

This specification is a product of the AToM MIB Working Group. 


\section{References}

[1] Case, J., McCloghrie, K., Rose, M., and S. Waldbusser, "Structure of Management Information for version 2 of the Simple Network Management Protocol (SNMPv2)", RFC 1442, SNMP Research, Inc., Hughes LAN Systems, Dover Beach Consulting, Inc., Carnegie Mellon University, April 1993.

[2] Case, J., McCloghrie, K., Rose, M., and S. Waldbusser, "Textual Conventions for version 2 of the the Simple Network Management Protocol (SNMPV2)", RFC 1443, SNMP Research, Inc., Hughes LAN Systems, Dover Beach Consulting, Inc., Carnegie Mellon University, April 1993.

[3] Case, J., McCloghrie, K., Rose, M., and S. Waldbusser, "Conformance Statements for version 2 of the the Simple Network Management Protocol (SNMPv2)", RFC 1444, SNMP Research, Inc., Hughes LAN Systems, Dover Beach Consulting, Inc., Carnegie Mellon University, April 1993.

[4] Galvin, J., and K. McCloghrie, "Administrative Model for version 2 of the Simple Network Management Protocol (SNMPv2)", RFC 1445, Trusted Information Systems, Hughes LAN Systems, April 1993.

[5] Case, J., McCloghrie, K., Rose, M., and S. Waldbusser, "Protocol Operations for version 2 of the Simple Network Management Protocol (SNMPV2)", RFC 1448, SNMP Research, Inc., Hughes LAN Systems, Dover Beach Consulting, Inc., Carnegie Mellon University, April 1993.

[6] McCloghrie, K., and M. Rose, "Management Information Base for Network Management of TCP/IP-based internets: MIB-II", STD 17, RFC 1213, Hughes LAN Systems, Inc., Performance Systems International, March 1991.

[7] Information processing systems - Open Systems Interconnection Specification of Abstract Syntax Notation One (ASN.1), International Organization for Standardization. International Standard 8824, (December, 1987).

[8] Information processing systems - Open systems Interconnection Specification of Basic Encoding Rules for Abstract Notation One (ASN.1), International Organization for Standardization. International Standard 8825, (December, 1987).

[9] American National Standard for Telecommunications - Digital Hierarchy - Optical Interface Rates and Formats Specification, ANSI T1.105-1988. 
[9a] ANSI T1.105-1991.

[10] American National Standard for Telecommunications - Digital Hierarchy - Optical Interface Specification (Single-Mode), ANSI T1.106-1988.

[11] American National Standard for Telecommunications -- Layer 1 InService Digital Transmission Performance Monitoring T1M1.3/93005R2, July 1993.

[12] McCloghrie, K., and F. Kastenholz, "Evolution of Interfaces Group of MIB-II", RFC 1573, Hughes LAN Systems, FTP Software, January 1994 .

[13] Cox, T., and K. Tesink, Editors, "Definitions of Managed Objects for the DS3/E3 Interface Type", RFC 1407, Bellcore, January 1993.

[14] Baker, F., and J. Watt, Editors, "Definitions of Managed Objects for the DS1/E1 Interface Type", RFC 1406, Advanced Computer Communications, Newbridge Networks Corporation, January 1993.

[15] CCITT Recommendation G.707, "Synchronous Digital Hierarchy Bit Rates", June 1992 .

[16] CCITT Recommendation G.708, "Network Node Interface for the Synchronous Digital Hierarchy", June 1992.

[17] CCITT Recommendation G.709, "Synchronous Multiplexing Structure", June 1992 .

[18] CCITT Recommendation G.783, "Characteristics of Synchronous Digital Hierarchy (SDH) Multiplexing Equipment Functional Blocks", November 1992. 
7. Security Considerations

Security issues are not discussed in this memo.

8. Authors' Addresses

Tracy A. Brown

Bell Communications Research

331 Newman Springs Road

P.O. Box 7020

Red Bank, NJ 07701-7020

Phone: (908) 758-2107

EMail: tacox@mail.bellcore.com

Kaj Tesink

Bell Communications Research

331 Newman Springs Road

P.O. Box 7020

Red Bank, NJ 07701-7020

Phone: (908) 758-5254

EMail: kaj@cc.bellcore.com 